\title{
1:1 Ground-track resonance in a uniformly rotating 4th degree and order gravitational field
}

\author{
Jinglang Feng ${ }^{1} \cdot$ Ron Noomen $^{1} \cdot$ Xiyun Hou $^{2} \cdot$ \\ Pieter Visser ${ }^{1}$. Jianping Yuan ${ }^{3}$
}

Received: 20 February 2016 / Revised: 11 June 2016 / Accepted: 19 July 2016 /

Published online: 12 September 2016

(C) The Author(s) 2016. This article is published with open access at Springerlink.com

\begin{abstract}
Using a gravitational field truncated at the 4th degree and order, the 1:1 groundtrack resonance is studied. To address the main properties of this resonance, a 1-degree of freedom (1-DOF) system is firstly studied. Equilibrium points (EPs), stability and resonance width are obtained. Different from previous studies, the inclusion of non-spherical terms higher than degree and order 2 introduces new phenomena. For a further study about this resonance, a 2-DOF model which includes a main resonance term (the 1-DOF system) and a perturbing resonance term is studied. With the aid of Poincare sections, the generation of chaos in the phase space is studied in detail by addressing the overlap process of these two resonances with arbitrary combinations of eccentricity $(e)$ and inclination $(i)$. Retrograde orbits, near circular orbits and near polar orbits are found to have better stability against the perturbation of the second resonance. The situations of complete chaos are estimated in the $e-i$ plane. By applying the maximum Lyapunov Characteristic Exponent (LCE), chaos is characterized quantitatively and similar conclusions can be achieved. This study is applied to three asteroids 1996 HW1, Vesta and Betulia, but the conclusions are not restricted to them.
\end{abstract}

Keywords Asteroid · Resonance width · Stability · Chaos · Poincaré sections · Equilibrium Points (EPs) · Vesta · Betulia · 1996 HW1

\section{Introduction}

The commensurability (usually a ratio of simple integers) between the rotation period of the primary body and the orbital period of the surrounding spacecraft or particle is called ground-track resonance. Many investigations have been carried out about geosynchronous

Jinglang Feng

J.Feng@tudelft.nl

1 Delft University of Technology, Kluyverweg 1, 2629 HS Delft, The Netherlands

2 Nanjing University, Nanjing, China

3 Northwestern Polytechnical University, Xi'an, China 
orbit, which is in 1:1 resonance with Earth. For example, a 2-DOF Hamiltonian was modeled by Delhaise and Henrard (1993) for the resonance near the critical inclination perturbed by the inhomogeneous geopotential. Global dynamics were studied in terms of Poincaré maps in the plane of inclination and argument of pericenter. Chaotic motions were expected close to the separatrix of the resonance of the mean motion.

However, for ground-track resonances in a highly irregular gravitational field (mainly small solar system bodies), the studies are limited. Scheeres (1994) studied the stability of the 1:1 ground-track resonance with a uniformly rotating asteroid using a triaxial ellipsoid model, and applied it to Vesta, Eros and Ida. Later on, he studied the effect of resonance between the rotation rate of asteroid Castalia and the true anomaly rate of an orbiting particle at periapsis with a 2nd degree and order gravitational field (Scheeres et al. 1996). This kind of resonance was proven to be responsible for significant changes of orbital energy and eccentricity, and provides a mechanism for an ejected particle to transfer into a hyperbolic orbit or vice versa. By considering the 2nd degree and order gravitational field, $\mathrm{Hu}$ and Scheeres (2004) showed that orbital resonance plays a significant role in determining the stability of orbits. Further, by modelling the resonant dynamics in a uniformly rotating 2 nd degree and order gravitational field as a 1-DOF pendulum Hamiltonian (Olsen 2006), the widths of the resonance were obtained in analytical expressions and also tested against numerical simulations for five resonances. They were found to be independent of the rotation rate and mass of the central body but strongly dependent on $e$ and $i$. The retrograde orbits have a smaller resonance region than the prograde ones. In a slowly rotating gravitational field, the orbital stability was explained by the distance between the resonances but not by the strength of a specific one using overlap criteria.

Recently, the resonant structure is further explored with the truncated model for the equatorial and circular cases, respectively. Delsate (2011) built the 1-DOF Hamiltonian of the ground-track resonances for Dawn orbiting Vesta. The locations of the EPs and the resonance width were obtained for several main resonances (1:1, 1:2, 2:3 and 3:2). The results were checked against numerical tests. The $1: 1$ and $2: 3$ resonances were found to be the largest and strongest one, respectively. The probability of capture in the 1:1 resonance and escape from it was found to rely on the resonant angle. Tzirti and Varvoglis (2014) extended Delsate's work by introducing $\mathrm{C}_{30}$ into the 1:1 resonance, which resulted in 2-DOF dynamics. The $\mathrm{C}_{30}$ term was found to create tiny chaotic layers around the separatrix but without significant influence on the resonance width. With the ellipsoid shape model (Compère et al. 2012), MEGNO (Mean Exponential Growth factor of Nearby Orbits) was applied as an indicator to detect stable resonant periodic orbits and also $1: 1$ and 2:1 resonance structures under different combinations of the three semi-major axes of the ellipsoid. A 1-DOF resonant model parametrized by $e$ and $i$ was obtained with a truncated ellipsoidal potential up to degree and order 2.

For previous studies listed above, the limitations are either the gravitational field which is truncated at degree and order 2 or the orbit which is restricted to a circular or polar case. In this study, the harmonic coefficients up to degree and order 4 are taken into account for studying the 1:1 resonance at different combinations of $e$ and $i$, which results in a 2-DOF model. More specifically, this paper is arranged as follows. Firstly, a 1-DOF Hamiltonian is built to investigate the main properties of the 1:1 resonance. The location of EPs and their stability are solved numerically for different combinations of $e$ and $i$ for Vesta, 1996 HW1 and Betulia. The resonance widths of the stable EPs are found numerically. Secondly, a 2-DOF Hamiltonian is introduced with the inclusion of a second resonance, which is treated as a perturbation on the 1-DOF Hamiltonian. Chaos is generated due to the overlap of the two resonances. By applying Poincaré sections, for all three asteroids, the extent of the chaotic 
region in the phase space is examined against the distance between the primary and second resonances and their respective strengths. The roles that $e$ and $i$ play on the evolution of chaos in the phase space are studied systematically. Finally, the maximal LCE (mLCE) of the orbits in the chaotic seas are calculated for a quantitative study.

\section{Dynamical modelling}

\subsection{Hamiltonian of the system}

The gravity potential expressed in orbital elements $(a, e, i, \Omega, \omega, M)$ is given by Kaula (1966) as

$$
V=\frac{\mu}{r}+\sum_{n \geq 2}^{\infty} \sum_{m=0}^{n} \sum_{p=0}^{n} \sum_{q=-\infty}^{\infty} \frac{\mu R_{e}^{n}}{a^{n+1}} F_{n m p}(i) G_{n p q}(e) S_{n m p q}(\omega, M, \Omega, \theta)
$$

in which $\mu$ and $R_{e}$ are the gravitational constant and reference radius of the body, respectively. $r$ Is the distance from the point of interest to the center of mass of the body. $F(i)$ and $G(e)$ are functions of inclination and eccentricity, respectively. The complete list of them up to degree and order 4 can be found in Kaula (1966) and Chao (2005). In addition, $n, m, p, q$ are all integers, $\theta$ is the sidereal angle

$$
S_{n m p q}=\left[\begin{array}{c}
C_{n m} \\
-S_{n m}
\end{array}\right]_{n-m \text { odd }}^{n-m \text { even }} \cos \Theta_{n m p q}+\left[\begin{array}{c}
S_{n m} \\
C_{n m}
\end{array}\right]_{n-m \text { odd }}^{n-m \text { even }} \sin \Theta_{n m p q},
$$

where $\Theta_{n m p q}$ is Kaula's phase angle, written as

$$
\Theta_{n m p q}=(n-2 p) \omega+(n-2 p+q) M+m(\Omega-\theta)
$$

Given the Delaunay variables

$$
\begin{gathered}
l=M, \quad g=\omega, \quad h=\Omega \\
L=\sqrt{\mu a}, \quad G=L \sqrt{\left(1-e^{2}\right)}, \quad H=G \cos i
\end{gathered}
$$

the Hamiltonian of the system can be written as

$$
\mathcal{H}=T-V+\dot{\theta} \Lambda
$$

in which $T=-\mu^{2} / 2 L^{2}$ is the kinetic energy and $\dot{\theta}$ is the rotation rate of the asteroid and $\Lambda$ is the momentum conjugate to $\theta$. Term $\theta \dot{\Lambda}$ appears due to the rotation of the asteroid (Delsate 2011). Resonances occur when the time derivative $\dot{\Theta}_{n m p q} \approx 0$. The 1:1 resonance is studied in detail in the following sections.

\subsection{1:1 Resonance}

According to Kaula (1966), to study the 1:1 resonance, the resonant angle is introduced and defined as $\sigma=\lambda-\theta$, with the mean longitude $\lambda=\omega+M+\Omega$. This resonance occurs at $\dot{\sigma} \approx 0$, which means that the revolution rate of the orbit equals the rotation rate of the asteroid. In addition, it should be noticed that the solution of this 1:1 resonance includes the equilibrium points (EPs) that are commonly studied in a rotating (or body-fixed) frame, which rotates with the asteroid and has its $z$-axis aligned with spin axis of the asteroid. The angle $\sigma$ represents the phase angle of the EPs in such a rotating frame. 
The spherical harmonics that contribute to this resonance are listed in Appendix 1. To introduce the resonant angle $\sigma$ in the Hamiltonian and also to keep the new variables canonical, a symplectic transformation is applied (Valk et al. 2009)

$$
d \sigma L^{\prime}+d \theta^{\prime} \Lambda^{\prime}=d \lambda L+d \theta \Lambda
$$

and a new set of canonical variables is obtained as

$$
\sigma, L^{\prime}=L, \theta^{\prime}=\theta, \Lambda^{\prime}=\Lambda+L
$$

After averaging over the fast variable $\theta^{\prime}$, the Hamiltonian for the 1:1 resonance truncated at the fourth order of $e$ can be written as

$$
\mathcal{H}=\mathcal{H}_{0}+\mathcal{H}_{1}+\mathcal{H}_{2}+o\left(e^{5}\right)
$$

where $\mathcal{H}_{k}(k=0,1,2)$ is the Hamiltonian that includes terms from the $k$ th order of $e$ in the $G(e)$ function. The lowest order in $\mathcal{H}_{0}, \mathcal{H}_{1}, \mathcal{H}_{2}$ is respectively $e^{0}, e^{1}, e^{2}$. In addition to these terms, there are still $e^{3}$ and $e^{4}$ terms included in the three Hamiltonians. Therefore, the remaining terms are $o\left(e^{5}\right)$. The expression of $\mathcal{H}_{0}$ is written as

$$
\begin{aligned}
\mathcal{H}_{0}= & -\frac{\mu^{2}}{2 L^{2}}+\dot{\theta}\left(\Lambda^{\prime}-L\right)-\frac{\mu^{4} R^{2}}{L^{6}}\left[C_{20}\left(1-e^{2}\right)\left(-\frac{3}{2}\right)\left(-\frac{1}{2}+\frac{3 s^{2}}{4}\right)\right. \\
& \left.+\frac{3}{4}\left(1-\frac{5 e^{2}}{2}+\frac{13 e^{4}}{16}\right)(1+c)^{2}\left(C_{22} \cos (2 \sigma)+S_{22} \sin (2 \sigma)\right)\right] \\
& -\frac{\mu^{5} R^{3}}{L^{8}}\left[\left(1+2 e^{2}+\frac{239 e^{4}}{64}\right)\left(-\frac{3}{4}(1+c)+\frac{15}{16}(1+3 c) s^{2}\right)\right. \\
& \times\left(C_{31} \cos \sigma+S_{31} \sin \sigma\right) \\
& \left.+\frac{15}{8}\left(1-6 e^{2}+\frac{423 e^{4}}{64}\right)(1+c)^{3}\left(C_{33} \cos (3 \sigma)+S_{33} \sin (3 \sigma)\right)\right] \\
& -\frac{\mu^{6} R^{4}}{L^{10}}\left[C_{40}\left(1-e^{2}\right)\left(-\frac{7}{2}\right)\left(\frac{3}{8}-\frac{15 s^{2}}{8}+\frac{105 s^{4}}{64}\right)\right. \\
& +\left(1+e^{2}+\frac{65 e^{4}}{16}\right)\left(-\frac{15}{8}(1+c)^{2}+\frac{105}{8}\left(c+c^{2}\right) s^{2}\right) \\
& \times\left(C_{42} \cos (2 \sigma)+S_{42} \sin (2 \sigma)\right) \\
& \left.+\frac{105}{16}\left(1-11 e^{2}+\frac{199 e^{4}}{8}\right)(1+c)^{4}\left(C_{44} \cos (4 \sigma)+S_{44} \sin (4 \sigma)\right)\right]
\end{aligned}
$$

in which $c=\cos (i), s=\sin (i)$ and $L$ is used hereafter instead of $L^{\prime}$ for convenience. In terms of angular variables, it can be seen that $\mathcal{H}_{0}$ is only dependent on the angle $\sigma$. Since $\theta^{\prime}$ is implicit in $\mathcal{H}_{0}$, its conjugate $\Lambda^{\prime}$ is a constant and can be dropped. Similarly, $G$ and $H$, which are related to $e$ and $i$, are constant as $g, h$ are absent in $\mathcal{H}_{0}$. Therefore at a given combination of $e$ and $i, \mathcal{H}_{0}$ is actually a 1-DOF system and is integrable. However, $\mathcal{H}_{1}$ and $\mathcal{H}_{2}$ are functions of both $\sigma$ and $g$ and include angles $j \sigma+k g(j=1,2,3, k= \pm 1, \pm 2)$, and therefore are 2-DOF systems. Their expressions are given in Appendix 1 and they are both zero at $e=0$ or $i=0$.

Since the origin of our selected body-fixed frame is located at the center of mass of the asteroid and the axes are aligned with the principal moments of inertia of the asteroid, the $\mathrm{C}_{21}, \mathrm{~S}_{21}$ and $\mathrm{S}_{22}$ terms are all zero, leading to the fact that $\mathcal{H}_{1}$ actually start from the $\mu^{5} R^{3} e \cdot \mathrm{C}_{30} / L^{8}$ term. However, $\mathcal{H}_{2}$ begins with $\mu^{4} R^{2} e^{2} \cdot \mathrm{C}_{22} / L^{6}$ term. As $e$ is not limited to 
small values in current study, it is difficult to compare the magnitudes of $\mathcal{H}_{1}$ and $\mathcal{H}_{2}$ directly. As a result, numerical simulations is performed for wide ranges of $e$ and $i$. It is found that $\mathcal{H}_{1} \in \mathcal{O}\left(\epsilon^{3 / 2}\right), \mathcal{H}_{2} \in \mathcal{O}(\epsilon)$, where $\epsilon$ is the ordering parameter that indicates the relative magnitudes of $\mathcal{H}_{1}, \mathcal{H}_{2}$ to $\mathcal{H}_{0}$ respectively and ranges from $10^{-2}$ to $10^{-1}$ for the current study. Therefore, $\mathcal{H}_{0}$ with resonant angle $\sigma$ can be viewed as the primary resonance. $\mathcal{H}_{1}$ and $\mathcal{H}_{2}$ are the second resonances, which are expected to give rise to chaos.

\section{Primary resonance}

\subsection{EPs and resonance width}

Firstly, $\mathcal{H}_{0}$ is studied in detail. Its equilibria can be found by numerically solving

$$
\dot{\sigma}=\frac{\partial \mathcal{H}_{0}}{\partial L}=0, \quad \dot{L}=-\frac{\partial \mathcal{H}_{0}}{\partial \sigma}=0
$$

The linearized system is written as

$$
\left[\begin{array}{l}
d \dot{\sigma} \\
d \dot{L}
\end{array}\right]=\left[\begin{array}{cc}
\frac{\partial^{2} \mathcal{H}_{0}}{\partial L \partial \sigma} & \frac{\partial^{2} \mathcal{H}_{0}}{\partial L^{2}} \\
-\frac{\partial^{2} \mathcal{H}_{0}}{\partial \sigma^{2}} & -\frac{\partial^{2} \mathcal{H}_{0}}{\partial L \partial \sigma}
\end{array}\right]\left[\begin{array}{l}
d \sigma \\
d L
\end{array}\right] .
$$

The linear stability of an EP can be determined from the Jacobian matrix evaluated at the EP. The resonant frequency can be approximated at a stable EP $\left(\sigma_{s}, L_{s}\right)$ as $\sqrt{\left.\frac{\partial^{2} \mathcal{H}_{0}}{\partial L^{2}} \cdot \frac{\partial^{2} \mathcal{H}_{0}}{\partial \sigma^{2}}\right|_{\sigma_{s}, L_{s}}}$. Taking the Hamiltonian value corresponding to an unstable $\operatorname{EP}\left(\sigma_{u}, L_{u}\right)$, denoted as $\mathcal{H}_{u}$, its level curve on the phase map is actually the separatrix that divides the motion into libration and circulation regions (Morbidelli 2002). Along this curve, $L$ passes through its maximum $L_{\max }$ and also minimum $L_{\min }$ at $\sigma=\sigma_{s}$. The resonance width is then calculated as $\Delta L=L_{\max }-L_{\min }$ and is therefore only valid for the stable EPs.

\subsection{Numerical results}

In this section, the EPs, their stability and the resonance width of asteroids Vesta, 1996 HW1 and Betulia are studied. They are selected because the first two asteroids are representatives of regular and highly bifurcated bodies, respectively, while Betulia has a triangular shape leading to large 3rd degree and order harmonics. Their shape models together with dimensions are given in Appendix 2. The 4th degree and order spherical harmonics of the three asteroids are also listed in Appendix 2. It is noted here that all the angles except for inclination in this study are represented in radians. First, the dynamics due to the 2nd degree and order harmonics $\left(\mathrm{C}_{20}\right.$ and $\left.\mathrm{C}_{22}\right)$, hereafter denoted as $\mathcal{H}_{0 / 2 n d}$, is studied. After that, we proceed with including other harmonic terms and investigate their effects on the phase portrait.

\subsubsection{Vesta}

As already mentioned in Sect. 2.2, the 1:1 resonance actually corresponds to the position of the EPs in the rotating frame. Figure 1 gives the mean semi-major axis of the 1:1 resonance in the $e-i$ plane. The EPs with $\sigma=0$ are unstable, while the ones with $\sigma=\pi / 2$ are stable. Due to the symmetry of the 2 nd degree and order gravitational field, the location of the EPs is symmetric with respect to $i=90^{\circ}$ and is closer to Vesta when the orbit is polar. 

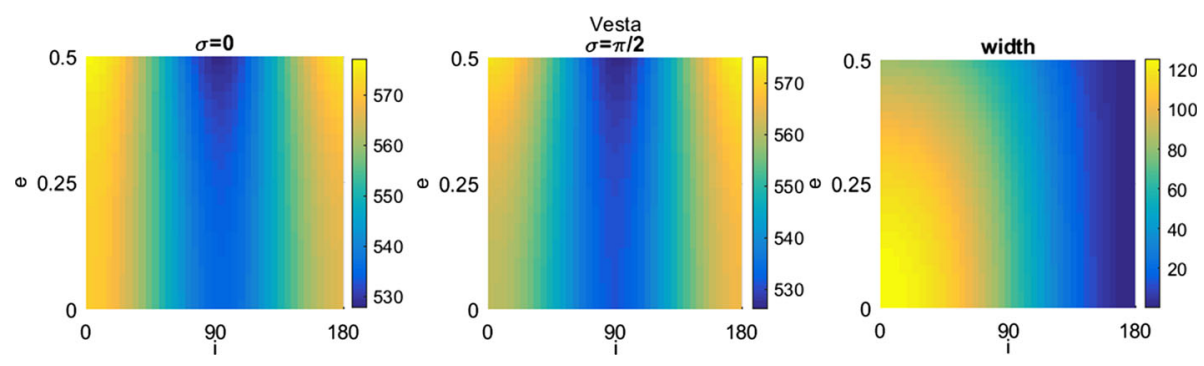

Fig. 1 The contour plots of mean semi-major axis (in km) of the unstable $(\sigma=0)$ and stable $(\sigma=\pi / 2) 1: 1$ resonance (the EPs) in the $e-i$ plane and the corresponding resonance width of stable EPs

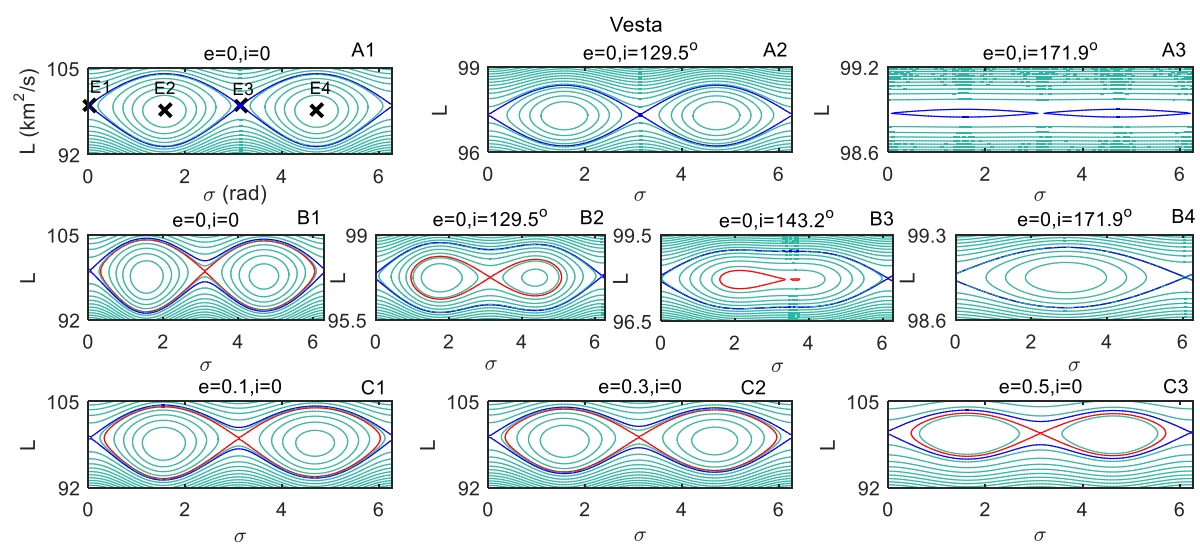

Fig. 2 The phase portrait of the Hamiltonian of Vesta. Top row $\mathcal{H}_{0 / 2 \text { nd }}$ for $e=0, i=0,129.5^{\circ}, 171.9^{\circ}$; middle row $\mathcal{H}_{0}$ for $e=0, i=0,129.5^{\circ}, 143.2^{\circ}, 171.9^{\circ}$; bottom row $\mathcal{H}_{0}$ for $i=0, e=0.1,0.3,0.5$. The blue and red lines are the separatrix (or the values of the Hamiltonian) of the unstable EPs. The conversion factor between $L^{2}$ and $a$ is $L^{2} / a=17.3 \mathrm{~km}^{3} / \mathrm{s}^{2}$

It gradually moves further away from Vesta when the orbit approaches the equatorial plane (either prograde or retrograde). The resonance width decreases with the increase of $i$, and finally becomes zero when $i$ arrives at $\pi$. This can be explained by the coefficient of the resonant angle $2 \sigma$ in Eq. (3), denoted as $f_{22}$

$$
f_{22}=-\frac{3 \mu^{4} R^{2}}{4 L^{6}} \cdot\left(1-\frac{5 e^{2}}{2}+\frac{13 e^{4}}{16}\right) \cdot(1+c)^{2} .
$$

When $i$ approaches $180^{\circ}$, the term $1+c$ becomes zero and $f_{22}$ also comes to zero. For a given value of $i$, the larger $e$ the smaller $f_{22}$ is and the resonance width also decreases (Fig. 1). However, this phenomenon is weakened for larger $i$ as its weight factor $(1+c)^{2}$ becomes smaller. This can clearly be observed from the contour map. In addition, our results for orbits at $e=0, i=0$ or $e=0, i=90^{\circ}$ are identical to those obtained in Delsate's study (Delsate 2011).

To investigate the effects of higher-order terms, the Hamiltonian $\mathcal{H}_{0}$ given in Eq. (3) is studied. Firstly, the phase portrait for some example orbits with different $e$ and $i$ is given in Fig. 2. The four EPs are marked out as E1, E2, E3 and E4. The sub-plots A1, A2 and A3 are actually the phase portrait of $\mathcal{H}_{0 / 2 n d}$ for comparison, and the remaining ones are those of $\mathcal{H}_{0}$. It can be seen that due to the inclusion of $3^{\text {rd }}$ and 4 th order harmonics, the symmetry 


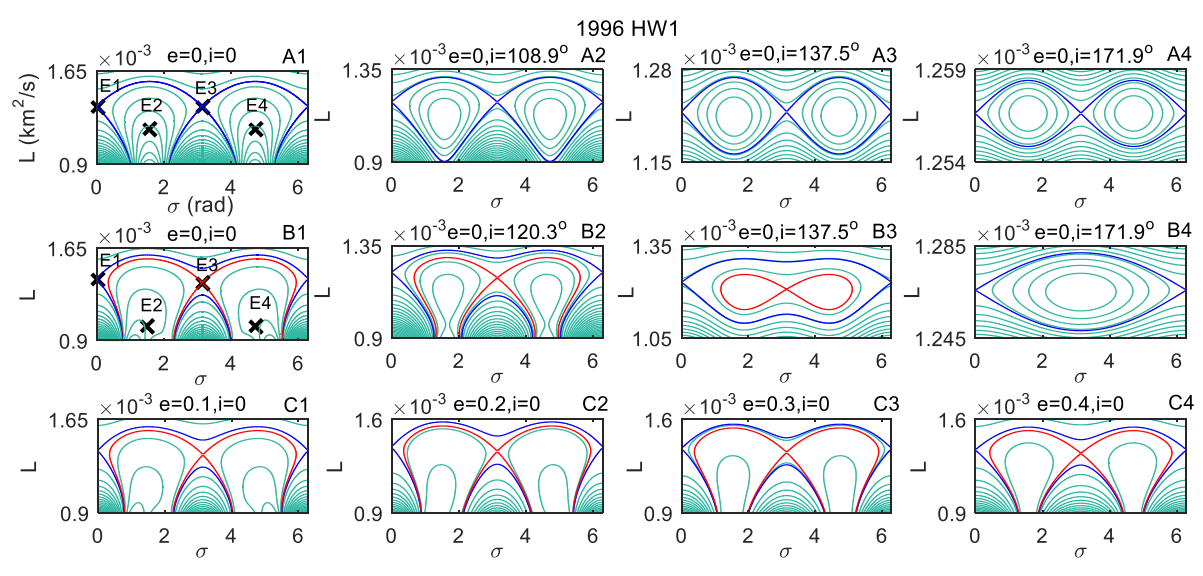

Fig. 3 The phase portrait of the Hamiltonian of 1996 HW1. Top row $\mathcal{H}_{0 / 2 n d}$ for $e=0, i=$ $0,108.9^{\circ}, 137.5^{\circ}, 171.9^{\circ}$; middle row $\mathcal{H}_{0}$ for $e=0, i=0,120.3^{\circ}, 137.5^{\circ}, 171.9^{\circ}$; bottom row $\mathcal{H}_{0}$ for $i=0, e=0.1,0.2,0.3,0.4$. The blue and red lines are the separatrix of the unstable EPs. The conversion factor between $L^{2}$ and $a$ is $L^{2} / a=5.8 \times 10^{-7} \mathrm{~km}^{3} / \mathrm{s}^{2}$

with respect to $\sigma=\pi$ is broken and the EPs have a shift from $\sigma=0$ and $\sigma=\pi / 2$ but are still located in the near vicinity of them. For the subplots B1-B4, with the increase of $i$, the two stable EPs gradually merge into one and the unstable EP around $\sigma=\pi$ disappears, as a result of the increasing strength of harmonic coefficients other than $\mathrm{C}_{22}$. The coefficient of the $\mathrm{C}_{31}$ term in Eq. (3) (denoted as $f_{31}$ ) is a first-order expression of $1+c$ while for that of $\mathrm{C}_{22}$ it is of the second order $(1+c)^{2}$ :

$$
f_{31}=-\frac{\mu^{5} R^{3}}{L^{8}} \cdot\left(1+2 e^{2}+\frac{239 e^{4}}{64}\right) \cdot\left(-\frac{3}{4}(1+c)+\frac{15}{16}(1+3 c) s^{2}\right) .
$$

When $i$ is large enough, the influence of $\mathrm{C}_{31}$ on the structure of the phase space exceeds that of $\mathrm{C}_{22}$. Therefore the phase space is dominated by the phase angle $\sigma$ of $\mathrm{C}_{31}$, and the existence of only one stable EP can be easily understood. In addition, all four EPs disappear when $i$ approaches $\pi$, which is due to the fact that all the coefficients of $k \sigma(k=1,2,3,4)$ in Eq. (3) become zero when $i=180^{\circ}$ because of the terms $1+c$ and $s^{2}$, and the phase portrait is filled with straight lines. The transit inclination (from four EPs to two EPs) is approximately $143^{\circ}$ at $e=0$ and slightly decreases to $126^{\circ}$ at $e=0.5$. This is explained by the fact that the $\mathrm{C}_{31}$ dynamics is strengthened when $e$ becomes larger, which is witnessed by the fact that the large $e$ promotes the merger of the two stable EPs shown in the C1-C3 sub-plots of Fig. 2. In addition, the larger the eccentricity, the smaller the value of the resonance width as predicted by the expression of $f_{22}$. Similarly, the larger the inclination $i$, the larger the resonance width as explained by the expression $f_{31}$ of the dominant coefficient, i.e. $\mathrm{C}_{31}$. The exact $\sigma$ values of the EPs and the resonance width are given as a contour map in the $e-i$ plane in Appendix 3.

\subsection{2 $1996 \mathrm{HWI}$}

For $1996 \mathrm{HW} 1$, the phase portrait of $\mathcal{H}_{0 / 2 n d}$ and $\mathcal{H}_{0}$ is given in Fig. 3. There are four unstable EPs appearing in the equatorial plane $(i=0)$, which is consistent with previous studies (Magri et al. 2011; Feng et al. 2015b). They are also marked as E1, E2, E3 and E4. For small $i$, there is no region for libration and therefore all the EPs are unstable. It can be 


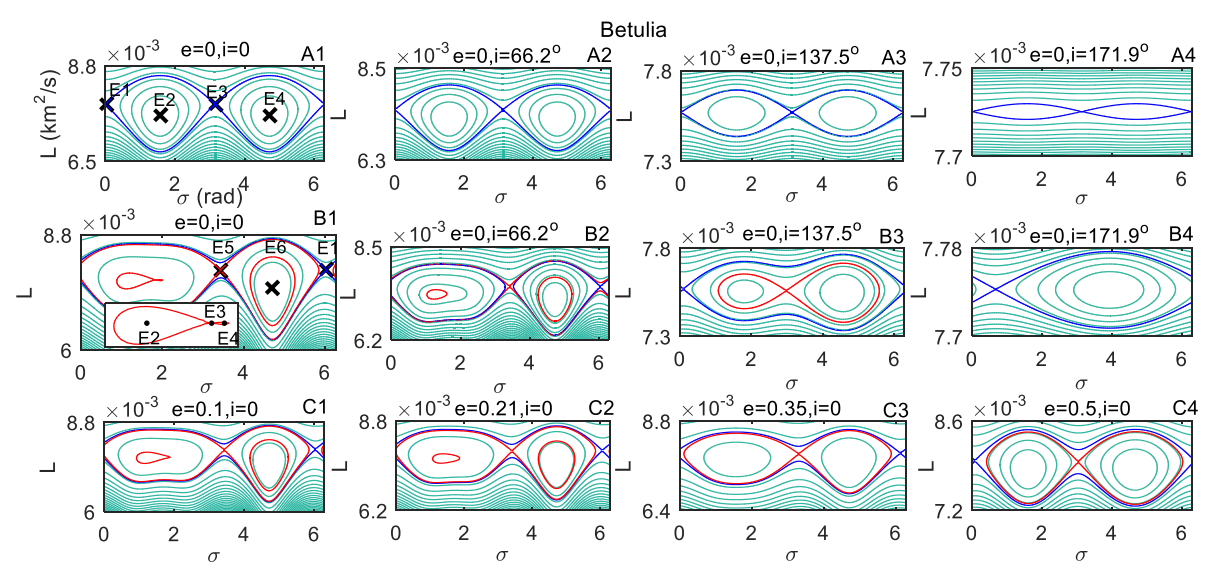

Fig. 4 The phase portrait of the Hamiltonian of Betulia including 4 th degree and order harmonics. Top $\mathcal{H}_{0 / 2 n d}$ for $e=0, i=0,66.2^{\circ}, 137.5^{\circ}, 171.9^{\circ}$; middle $\mathcal{H}_{0}$ for $e=0, i=0,66.2^{\circ}, 137.5^{\circ}, 171.9^{\circ}$; bottom $\mathcal{H}_{0}$ for $i=0, e=0.1,0.21,0.35,0.5$. The blue and red lines are the separatrix of the unstable EPs. The conversion factor between $L^{2}$ and $a$ is $L^{2} / a=1.1 \times 10^{-5} \mathrm{~km}^{3} / \mathrm{s}^{2}$

seen that the instability of the four EPs is already determined by the dynamics of $\mathcal{H}_{0 / 2 n d}$. The inclusion of other harmonics, however, causes a strong distortion of the phase space. Two of the unstable EPs become stable at $i \approx 108.9^{\circ}$ (Fig. 3A2) for $\mathcal{H}_{0 / 2 n d}$ and at $i \approx 120.3^{\circ}$ (Fig. 3B2) for $\mathcal{H}_{0}$, indicating the destabilizing effects of the highly irregular gravitational field and the stability of the retrograde motion in this highly perturbed environment. Then, the two EPs merge into one at $i \approx 154.7^{\circ}$ because of the $\mathrm{C}_{31}$ term, and finally disappear due to the $(1+c)$ and $s^{2}$ terms in $f_{31}$. The phase portrait is also slightly influenced by $e$, and similarly the larger eccentricity the larger effects it has on the phase portrait in terms of resonance width, as shown in the subplots Fig. 3C1-C4.

The $\sigma$ of the EPs and the resonance width are only obtained for the situation where stable EPs exist and are given in Appendix 3. The semi-major axis of the stable EPs and the unstable EPs are also given, indicated by $\mathrm{a}_{\mathrm{s}}$ and $\mathrm{a}_{\mathrm{u}}$, respectively. Observe that after arriving at the maximum value at $\mathrm{i} \approx 128.9^{\circ}$, the resonance width decreases and becomes zero when i approaches $180^{\circ}$. However, it is not affected by e, as the dynamics is mainly dominated by $i$ rather than $e$. Therefore the most interesting range for resonance is within $126^{\circ}<i<171.9^{\circ}$, and it will be further studied in the next section.

\subsubsection{Betulia}

The phase portrait of Betulia is given in Fig. 4. Only four EPs (E1, E2, E3 and E4) appear for $\mathcal{H}_{0 / 2 n d}$, while there are six EPs apparent in the equatorial plane for $\mathcal{H}_{0}$ due to the triangular shape of this body. Among them, E2, E4 and E6 are stable and E1, E3 and E5 are unstable. In Magri's study (2007), six EPs were also obtained near the equatorial plane of Betulia, but using a polyhedron gravitational field. However, in that paper the authors find E6 to be unstable, whereas it appears to be stable according to our phase portrait (right stretched island in Fig. 4B1). The second difference is that their EPs are in general slightly closer to the body than ours. These distinctions primarily originate from the different gravitational fields applied in their work and the current study. Although EP6 was not originally detected as being unstable, however, the gravitational field truncated at degree and order 4 applied in 
this study captures the major dynamical properties of the complete gravitational field for the 1:1 resonance, in terms of the number and approximate location of the EPs.

As shown in the sub-plots B1-B4 of Fig. 4, the phase portrait of $\mathcal{H}_{0}$ changes significantly with the increase of $i$. There are actually three EPs within the left main island, which are illustrated as E2, E3 and E4 in the plot. Among them, E2 and E4 are stable, while E3 is unstable and the most inner red line is its separatrix. From $i \approx 66.2^{\circ}$, the unstable EP disappears and the two stable ones start to merge, as shown in Fig. 4B3 with only two stable EPs left. Similarly, when $i$ gets close to $180^{\circ}$, only one EP exists due to the dominant effect of $C_{31}$. In addition, because of the triangular shape of Betulia, the $C_{31}, S_{31}$ and $S_{33}$ terms are large compared to other asteroids, e.g. Vesta and $1996 \mathrm{HW} 1$. Although $\mathrm{S}_{33}$ is one order of magnitude smaller than $\mathrm{C}_{22}$, the coefficients of their phase angles $3 \sigma$ and $2 \sigma$ respectively are comparable with each other for small $e$ and $i$. As such, it is the $\mathrm{S}_{33}$ term that introduces two more EPs and also makes the phase space asymmetric with respect to $\sigma=\pi$. With the increase of both $e$ and especially $i$, the influence of $\mathrm{C}_{31}$ becomes much stronger than that of $\mathrm{C}_{22}$ and $\mathrm{S}_{33}$ and finally dominates the phase space as in the previous study cases. In addition, the right island where EP6 is located is always slightly larger than the left one. In the next section, the resonance width of Betulia is actually measured as the width of the larger one. The exact values of $\sigma$ of the EPs, the corresponding semi-major axes, and the resonance width are also given in Appendix 3.

In summary, the effects of $i, e$ and the spherical harmonics on the evolution of the phase portrait of the 1:1 ground track resonance have been studied through the 1-DOF Hamiltonian $\mathcal{H}_{0}$. Due to the $1+c$ term in the coefficients of all phase angles of $\mathcal{H}_{0}$, the resonance width of the retrograde orbits is found to be smaller than that of the prograde ones, which is consistent with Olsen's conclusion (2006). The eccentricity also affects the phase space in terms of resonance width and the number of EPs. Although it is found that the stability of the EPs is largely determined by the 2nd degree and order harmonics, especially for Vesta and 1996 HW1, the strength of $\mathrm{C}_{31}$ exceeds $\mathrm{C}_{22}$ and dominates the structure of the phase space when $i$ approaches $180^{\circ}$. The large $\mathrm{S}_{33}$ term not only brings about more EPs but also introduces significant asymmetry of the phase space with respect to $\sigma=\pi$.

\section{Second resonance}

For a more complete analysis of the 1:1 resonant dynamics, in addition to $\mathcal{H}_{0}, \mathcal{H}_{1}$ and $\mathcal{H}_{2}$ should also be considered, which introduce one more degree of freedom in the dynamics. However, the inclusion of all terms in $\mathcal{H}_{1}$ and $\mathcal{H}_{2}$ is far from trivial. For this study, the dominant term of $\mathcal{H}_{1}$ and $\mathcal{H}_{2}$ is taken into account and is treated as a second resonance. The dominant term, which has the largest amplitude, is given by

$$
\mathcal{H}_{2 d}=-\frac{\mu^{4} R^{2}}{L^{6}}\left[F_{221} G_{212}\left(C_{22} \cos (2 \sigma-2 g)+S_{22} \sin (2 \sigma-2 g)\right)\right]
$$

Therefore, the resulting 2-DOF Hamiltonian is written as

$$
\mathcal{H}_{2 \text { dof }}=\mathcal{H}_{0}+\mathcal{H}_{2 d}
$$

A new resonant angle $2 \sigma-2 g$ is introduced in the dynamics in addition to $k \sigma(k=1,2,3,4)$. A formal way to deal with this system is to treat $\mathcal{H}_{2 d}$ as a small perturbation to the integrable system $\mathcal{H}_{2 \text { dof }}=\mathcal{H}_{0}$ (Henrard 1990). However, in our study, the perturbation from $\mathcal{H}_{2 d}$ is not necessarily to be small values, due to the large variations of $e$ and $i$. 
According to Chirikov (1979) and Morbidelli (2002), the dynamics of $\mathcal{H}_{2 \text { dof }}$ can be studied by observing the overlap process of nearby resonances using Poincaré maps. To a first approximation, each resonance is considered separately, namely only its own resonant angle is taken into account and the other one is neglected. The first resonance $\mathcal{H}_{\text {reson } 1}$ is actually $\mathcal{H}_{0}$, and the second resonance $\mathcal{H}_{\text {reson } 2}$ is defined as

$$
\begin{aligned}
\mathcal{H}_{\text {reson } 2}= & -\frac{\mu^{2}}{2 L^{2}}-\dot{\theta} L-\frac{\mu^{4} R^{2}}{L^{6}} F_{201} G_{210} C_{20}+\mathcal{H}_{2 d} \\
= & -\frac{\mu^{2}}{2 L^{2}}-\dot{\theta} L-\frac{\mu^{4} R^{2}}{L^{6}}\left(1-e^{2}\right)\left(-\frac{3}{2}\right)\left(-\frac{1}{2}+\frac{3 s^{2}}{4}\right) C_{20} \\
& -\frac{\mu^{4} R^{2}}{L^{6}} \frac{3}{2}\left(\frac{9 e^{2}}{4}+\frac{7 e^{4}}{4}\right) s^{2}\left[C_{22} \cos (2 \sigma-2 g)+S_{22} \sin (2 \sigma-2 g)\right]
\end{aligned}
$$

which only includes one resonant angle $2 \sigma-2 g$. Their locations need to be solved first and then the Poincaré maps of the single-resonance dynamics can be computed on a common surface of section in the vicinity of their location. If $\mathcal{H}_{2 d}$ is small, the separatrix of $\mathcal{H}_{\text {reson } 2}$ is

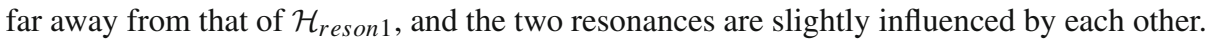
Tiny chaotic layers are probably generated around the separatrix. Otherwise, if $\mathcal{H}_{2 d}$ is large enough, the separatrix of the two resonances intersect, their dynamical domains overlap, and each resonance is significantly influenced by the other one. The chaotic layers extend to large-region chaos that dominates the phase space. Since $\mathcal{H}_{\text {reson } 1}$ is the dominant dynamics of our 1:1 resonant model, the focus is put on how $\mathcal{H}_{\text {reson } 1}$ is influenced by $\mathcal{H}_{\text {reson } 2}$, which can also be interpreted as how much the 1-DOF dynamics is affected by a perturbation.

\subsection{The location and width of $\mathrm{H}_{\text {reson2 }}$}

The location and width of $\mathcal{H}_{\text {reson } 1}$ have been obtained in Sect. 3. Since we want to apply Poincare sections to study the dynamics, the section of the map is first defined here as $g=\pi / 2, \dot{g}>0$ in the $L-\sigma$ plane. Namely, when integrating the 2-DOF Hamiltonian, when the solution crosses this section, its state is recorded on the $L-\sigma$ plane. Since $\mathcal{H}_{\text {reson } 1}$ has 1-DOF, its Poincaré map is the same with its phase portrait in the phase space. The location of $\mathcal{H}_{\text {reson } 2}$ on this section can be obtained by numerically solving

$$
\left\{\begin{array}{l}
2 \dot{\sigma}-2 \dot{g}=\frac{2 \partial \mathcal{H}_{\text {reson } 2}}{\partial L}-\frac{2 \partial \mathcal{H}_{\text {reson } 2}}{\partial G}=0 \\
\mathcal{H}_{\text {reson } 2}\left(\sigma_{0}, g_{0}, L^{*}, G^{*}\right)=\mathcal{H}_{\text {separatrix }}
\end{array}\right.
$$

in which $\sigma_{0}=g_{0}=\pi / 2 . \mathcal{H}_{\text {seperatrix }}$ is the Hamiltonian value of the separatrix of $\mathcal{H}_{\text {reson } 1}$ which is also the energy constant of the section. $L^{*}$ and $G^{*}$ represent the variables that need to be solved. As $\mathcal{H}_{\text {reson } 2}$ itself is a 2-DOF system, the pendulum model cannot be applied for approximating its resonance width. Therefore, based on the dynamical properties of the Poincaré map, a full numerical estimation is used. By integrating from the initial point $\left(\sigma_{0}, g_{0}, L^{*}, G^{*}\right)$ over a proper time duration, a curve is obtained which is either the upper or the lower part of the separatrix of $\mathcal{H}_{\text {reson } 2}$ on the section. If it is the upper part, $L_{\max }$ is directly obtained by taking record of the maximum point of the curve. $L_{\min }$ is the minimum of the lower border obtained by integrating from the point $\left(\sigma_{0}, g_{0}, L^{*}-\delta L, G\right)$ with a displacement from $\left(\sigma_{0}, g_{0}, L^{*}, G^{*}\right)$ by $\delta L$ depending on the dynamics studied and vice versa. The curves acquired are the separatrix of $\mathcal{H}_{\text {reson } 2}$. Therefore, the width of $\mathcal{H}_{\text {reson } 2}$ is approximated by $L_{\max }-L_{\min }$, which is already good enough for the current study.

Given that the maxima and minima of $\mathcal{H}_{\text {reson } 1}$ and $\mathcal{H}_{\text {reson } 2}$ are denoted as $L_{\max 1}, L_{\min 1}$ and $L_{\max 2}, L_{\min 2}$, respectively, the relative locations of the two resonances can be character- 

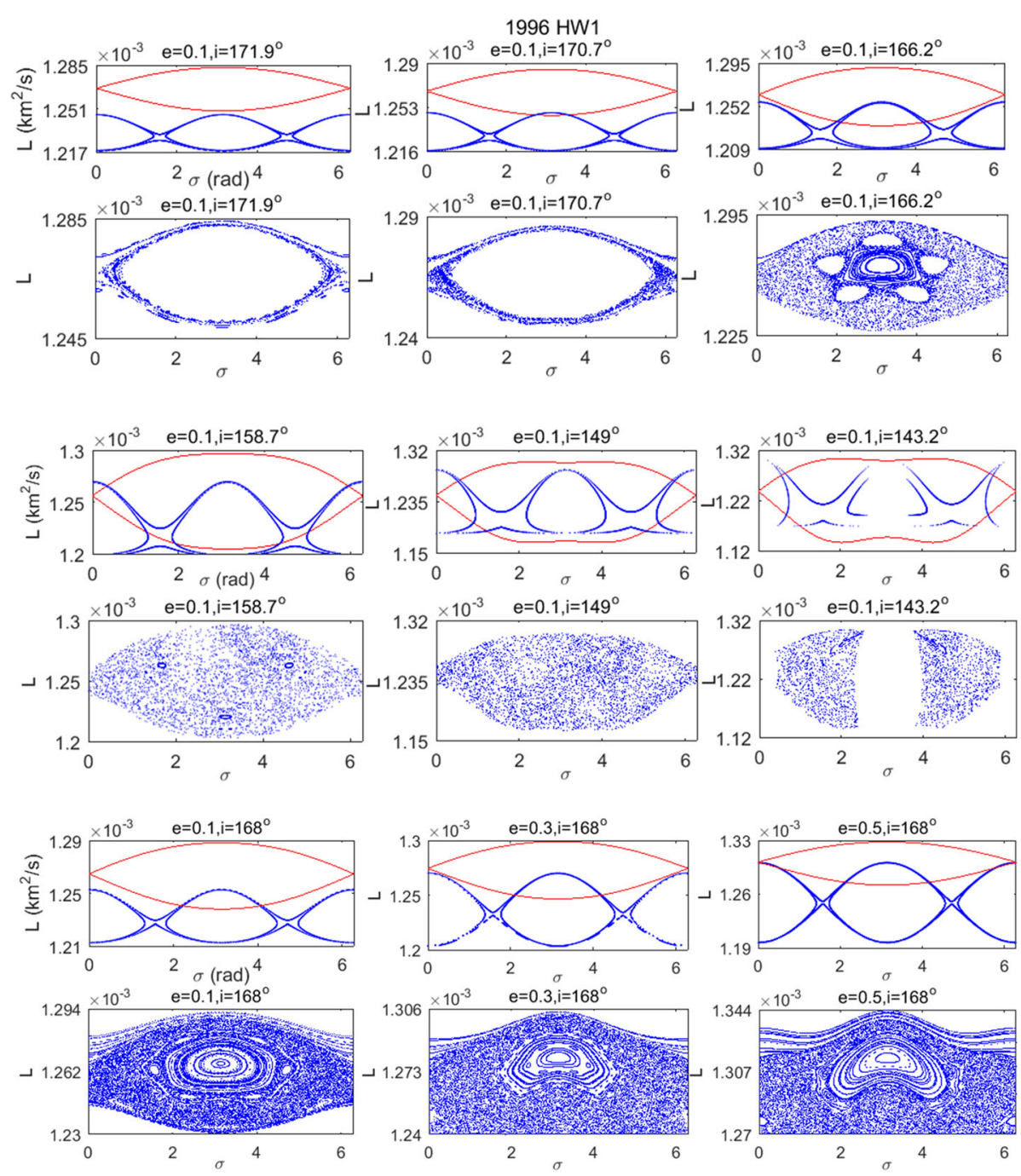

Fig. 5 First and third rows: the separatrices of resonances $\mathcal{H}_{\text {reson } 1}($ red $)$ and $\mathcal{H}_{\text {reson } 2}$ (blue) on the section $g=\pi / 2, \dot{g}>0$; second and fourth rows: the phase space of the corresponding $\mathcal{H}_{2 d o f}$; both for $e=0.1, i=$ $171.9^{\circ}, 170.7^{\circ}, 166.2^{\circ}, 158.7^{\circ}, 149^{\circ}, 143.2^{\circ}$. Fifth and sixth rows: the separatrices of $\mathcal{H}_{\text {reson } 1}$ (red) and $\mathcal{H}_{\text {reson } 2}$ (blue) and the corresponding $\mathcal{H}_{2 \text { dof }}$ for $i=168^{\circ}, e=0.1, .3, .5$

ized by $L_{\min 1}-L_{\max 2}$ and $L_{\min 1}-L_{\min 2}$. The former one, which is the distance between the closer borders of the two resonances, is positive if the two resonances are totally separated and becomes negative as the resonances start to overlap with each other. The latter one is actually the measurement of the extent of overlap of the two resonances. Its non-positive value indicates that one resonance is completely within the other one. For different combinations of $e$ and $i$, the 2-DOF Hamiltonian $\mathcal{H}_{2}$ dof is studied for $1996 \mathrm{HW} 1$, Vesta and Betulia.

\subsection{HW1}

Since $1996 \mathrm{HW} 1$ has a limited inclination range $\left(126^{\circ} \lesssim i<180^{\circ}\right)$ of libration motion of $\mathcal{H}_{\text {reson } 1}$ (shown in Fig. 3), its second degree of freedom dynamics is studied first. In Fig. 5, 
the upper plots give the separatrices of the two resonances on the Poincare maps, which are the boundaries of their phase space. The bottom plots are the phase space of $\mathcal{H}_{2 \text { dof }}$ on the same section, both for $i$ changing from $171.9^{\circ}$ to $143.2^{\circ}$ at the example $e=0.1$. Fig. 5 reflects the relationship of the distance between the two resonances $\mathcal{H}_{\text {reson } 1}$ and $\mathcal{H}_{\text {reson } 2}$ and the extent of chaotic region of $\mathcal{H}_{2 \text { dof }}$.

\subsubsection{The effect of $i$}

For $i=171.9^{\circ}$, even though the resonances do not overlap (but are close), tiny chaotic layers appear in the vicinity of the separatrix of $\mathcal{H}_{2 d o f}$. When there is a small overlap at $i=166.2^{\circ}$, the chaotic layer is extended but a large libration region still remains. With the increase of the overlap from $i=166.2^{\circ}$, a large part of the phase space is occupied by chaos. The regular region shrinks to a limited area at the center of the phase space and meanwhile five islands appear around it, which is due to the high-order resonances between $\mathcal{H}_{\text {reson } 1}$ and $\mathcal{H}_{\text {reson } 2 .}$ With the further decrease of $i$ to $158.7^{\circ}, \mathcal{H}_{\text {reson } 2}$ is almost completely inside $\mathcal{H}_{\text {reson } 1}$ and there are only three small KAM tori left, indicating that the system is transiting to global chaos. In addition, the original stable EP becomes unstable as the center part is already chaotic. Although the dynamics is completely chaotic at $i=149^{\circ}$, the chaos is still bounded by the separatrix of $\mathcal{H}_{\text {reson } 1}$. However, finally at $i=143.2^{\circ}$ the whole structure of $\mathcal{H}_{\text {reson } 1}$ could not be kept and the continuity of phase space is broken. It is noticed that this break is consistent with the break of the separatrix of $\mathcal{H}_{\text {reson } 2}$ at the same range of $\sigma$, implying a significant perturbation of $\mathcal{H}_{\text {reson } 2}$ on the total dynamics. The break of $\mathcal{H}_{\text {reson } 2 \text { 's separatrix attributes }}$ to the fact that the time derivative of $g$ (namely $\dot{g}$ ) changes its sign from positive to negative after $i$ crossing some specific value, and therefore it produces no crossings on the section which is defined as $\dot{g}>0$. This phenomenon will be discussed in detail in the next section.

In summary, $i$ has a significant influence on the 2-DOF dynamics at constant $e$. When $i$ decreases, $\mathcal{H}_{\text {reson } 2}$ is strengthened as it includes the term $s^{2}$ (as seen in Eq. 6) and its resonance width increases. However, its location does not deviate much. For $\mathcal{H}_{\text {reson } 1}$, not only its width is increasing but also its location is moving downward. Ultimately, the two resonances totally overlap and have a strong interaction with each other. Nevertheless, the width of $\mathcal{H}_{2 \text { dof }}$ is determined by $\mathcal{H}_{\text {reson } 1}$, because the $L_{\max }$ and $L_{\min }$ values of the former match the ones of the latter, although the inner structure of the phase space has been totally affected.

\subsubsection{The effect of $e$}

To study the effect of $e$ on the dynamics, the contour map of the distance of the two resonances and also the width of the second resonance are given on the $e-i$ plane in Fig. 6. In the left plot, the yellow region indicates the situation of non-overlap and slight overlap. In the middle plot, the green and blue areas demonstrate the situation when $\mathcal{H}_{\text {reson } 2}$ moves totally inside $\mathcal{H}_{\text {reson } 1}$ and the overlap between the two is complete. The right plot demonstrates that the width of $\mathcal{H}_{\text {reson } 2}$ is also enlarged when $e$ becomes large, which can be proven by the term $\left(9 e^{2} / 4+7 e^{4} / 4\right)$ in $\mathcal{H}_{\text {reson } 2}$. Therefore, the largest distance of $\mathcal{H}_{\text {reson } 1}$ and $\mathcal{H}_{\text {reson } 2}$ is observed at the down-right corner and $\mathcal{H}_{\text {reson } 2}$ approaches its highest location at the upper-left corner in the left plot. In addition, as $e$ increases and $i$ decreases, $\mathcal{H}_{\text {reson } 2}$ becomes stronger (as indicated by the resonance width) and has a significant influence on the dynamics of $\mathcal{H}_{\text {reson } 1}$.

Therefore, given a specific $e$ and $i$, an estimation from this contour map can be made on when small chaotic layers appear and when large chaotic seas are expected. As an example, for $e=0.1$, tiny chaotic layers are apparent at $i=171.9^{\circ}$ when the two resonances start to overlap; the last KAM tori disappear and the phase space is full with chaos around $i=158.2^{\circ}$. 

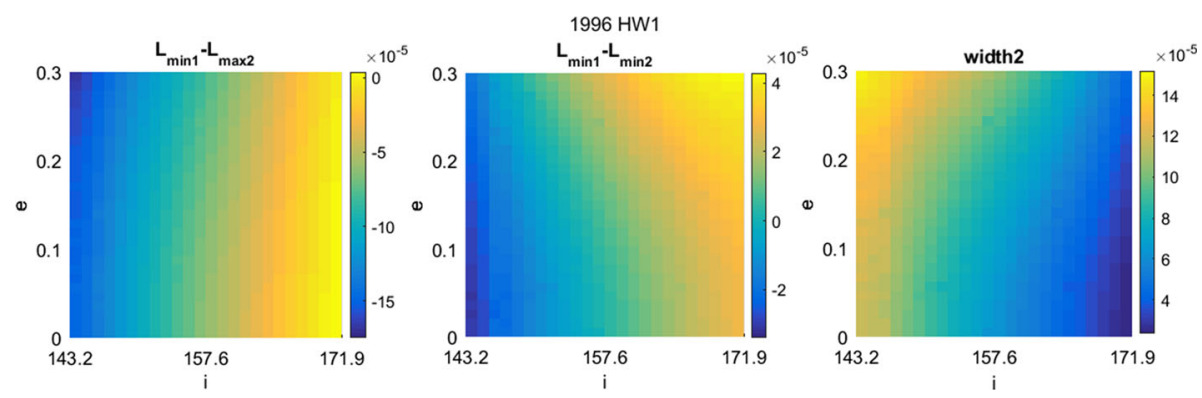

Fig. 6 The distance between $\mathcal{H}_{\text {reson } 1}$ and $\mathcal{H}_{\text {reson } 2}$ measured as $L_{\min 1}-L_{\max 2}$ (left) and $L_{\min 1}-L_{\min 2}$ (middle), and the width of $\mathcal{H}_{\text {reson } 2}$ (right)
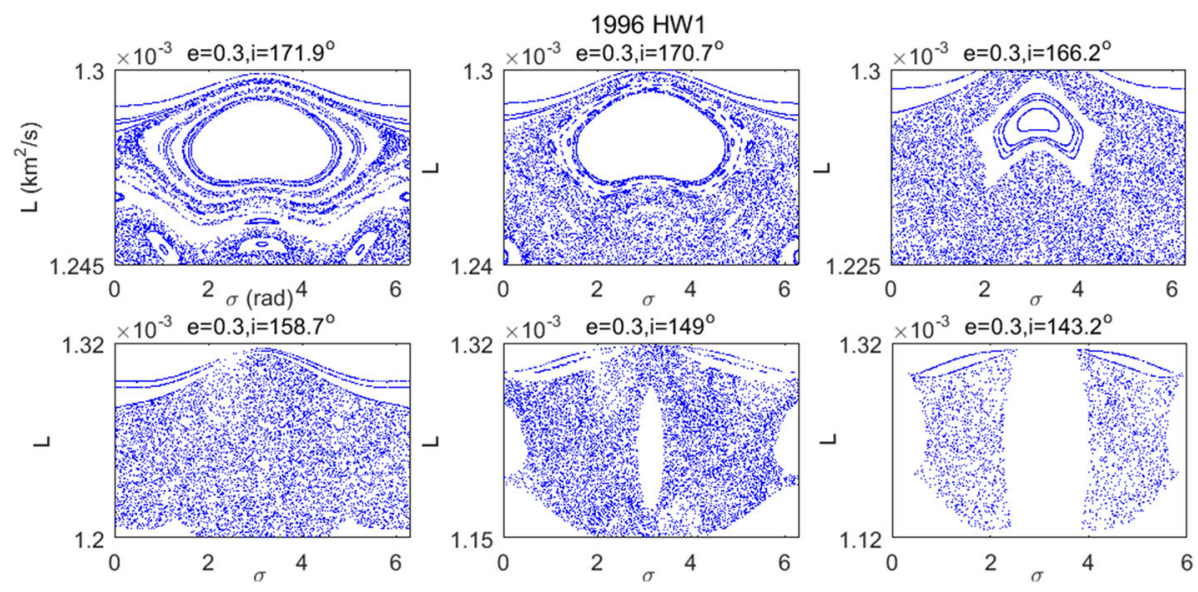

Fig. 7 The phase space of $\mathcal{H}_{2 \text { dof }}$ at $e=0.3$ for $i=171.9^{\circ}, 170.7^{\circ}, 166.2^{\circ}, 158.7^{\circ}, 149^{\circ}, 143.2^{\circ}$

For a more complete understanding, the phase space of $\mathcal{H}_{2 \text { dof }}$ at $e=0.3$ with different $i$ is given in Fig. 7. As compared to Fig. 5, the upper plots of Fig. 7 show that the large $e$ distorts the main island, which originally has a circular or ellipsoidal shape. The chaos is more abundant and the size of the main island reduces and a new phase structure is generated at the bottom of the plot, due to the stronger influence of $\mathcal{H}_{\text {reson } 2}$. In addition, the lower half of the chaos is thicker than the upper part, as it is more influenced by the perturbation from $\mathcal{H}_{\text {reson } 2}$ which approaches $\mathcal{H}_{\text {reson } 1}$ from the bottom direction. In addition, the islands appearing at the bottom area of the phase space can be explained by the direct interaction of $\mathcal{H}_{\text {reson } 1}$ and $\mathcal{H}_{\text {reson } 2}$ in that region. Furthermore, the lower three plots are full of chaos.

For $e=0.3, i=149^{\circ}, \mathcal{H}_{\text {reson } 2}$ is already comparable with $\mathcal{H}_{\text {reson } 1}$ on the dynamics of $\mathcal{H}_{2 \text { dof }}$. Therefore, the center part of the phase space is not completely chaotic; a limited regular (white) region appears which is actually part of the center island of $\mathcal{H}_{\text {reson } 2 .}$

\subsubsection{Bounded chaotic regions}

As mentioned in Sect. 4.2.1, tiny chaotic layers are generated in the vicinity of the separatrix. Their boundaries can be estimated, which is the topic of this section. 

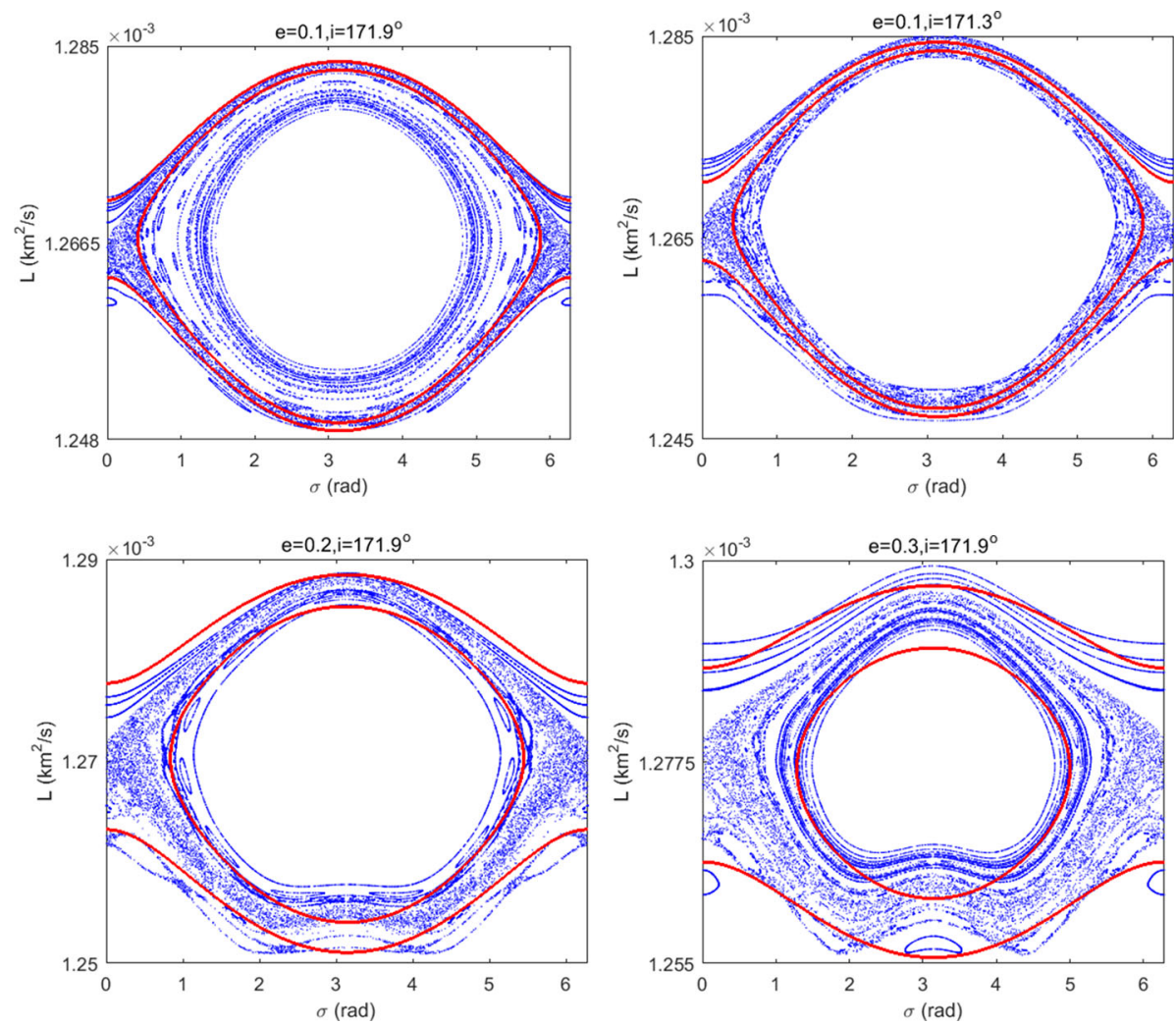

Fig. 8 The chaotic regions bounded by the separatrices (red lines) corresponding to the minimal and maximal resonant width for $e=0.1, i=171.9^{\circ}, 171.3^{\circ} ; i=171.9^{\circ}, e=0.2,0.3$

For small perturbations, it is known from Morbidelli (2002) that the chaotic region covers areas spanned by the instantaneous separatrices for varying secular angles, which is $g$ in our study. Its boundaries are estimated from the separatrices corresponding to the minimal and maximal resonant width. This is also known as the modulated-pendulum approximation. The small perturbation corresponds to the case of close approach and almost contact between $\mathcal{H}_{\text {reson } 1}$ and $\mathcal{H}_{\text {reson } 2}$, and is therefore applicable to a situation with quite large inclination values. For 1996 HW1, Fig. 8 illustrates this region at different eccentricities and inclinations.

In each plot, the outer red lines represent the boundary corresponding to the Hamiltonian value of $\mathcal{H}_{\text {separatrix }}+\mathcal{H}_{2 d}(g=0)$ which is the maximum resonance width. The inner red line is the inner boundary with the Hamiltonian value of $\mathcal{H}_{\text {separatrix }}+\mathcal{H}_{2 d}(g=\pi / 2)$ which is the minimum resonance width. For $e=0.1, i=171.9^{\circ}$, in which case the perturbation from $\mathcal{H}_{\text {reson } 2}$ is weakest (shown in Fig. 5), the theory works perfectly since all chaos is restricted to the region between the two red lines. When the inclination decreases to $171.3^{\circ}$, the chaos is still well bounded but a small portion of it at the bottom area is already outside the red lines. For comparison, the cases of $e=0.2, i=171.9^{\circ}$ and $e=0.3, i=171.9^{\circ}$ are studied, in which situation the perturbations of $\mathcal{H}_{\text {reson } 2}$ are not small anymore. The chaotic region does not fit within the red lines well. The bottom part of the chaos is shifted upwards and is therefore outside the inner red line, due to the distortion of the phase space. 


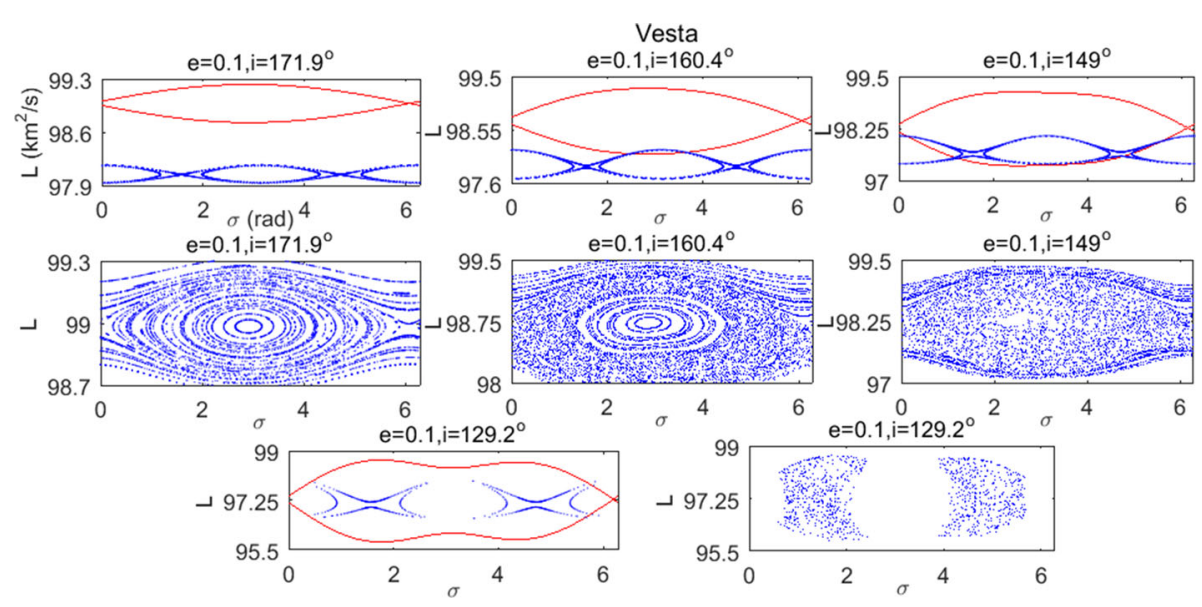

Fig. 9 First row the separatrices of resonances $\mathcal{H}_{\text {reson } 1}($ red $)$ and $\mathcal{H}_{\text {reson } 2}$ (blue) on the section $g=\pi / 2, \dot{g}>$ 0 ; second row the phase space of the corresponding $\mathcal{H}_{2 \text { dof }}$; both for $e=0.1, i=171.9^{\circ}, 160.4^{\circ}, 149^{\circ}$; third row the separatrices and phase space for $e=0.1, i=129.2^{\circ}$ (retrograde)

In summary, the chaotic layers are well estimated with the approximation theory for the small perturbation cases ( $i$ close to $\pi$ and small $e$ ). The strong perturbation of $\mathcal{H}_{\text {reson } 2}$ introduced by large $e$ not only broadens the chaotic region, but also reshapes the phase space.

\subsection{Vesta}

\subsubsection{The effect of $i$}

For Vesta, there is always a stable EP for different $i$ and $e$. For the retrograde case, the distance between $\mathcal{H}_{\text {reson } 1}$ and $\mathcal{H}_{\text {reson } 2}$ and the phase space of $\mathcal{H}_{2 \text { dof }}$ for orbits with different $i$ but the same $e=0.1$ is given in Fig. 9. For $i=171.9^{\circ}$, the dynamics of $\mathcal{H}_{\text {reson } 1}$ is hardly influenced since the two resonances are far apart and there is no interaction between them. With the decreasing of $i$, a significant chaotic region around the separatrix appears, even when the two resonances are just in contact (as seen at $i=160.4^{\circ}$ ). When $\mathcal{H}_{\text {reson } 2}$ completely evolves inside $\mathcal{H}_{\text {reson } 1}$, the phase space becomes totally chaotic, as indicated at $i=149^{\circ}$. Further at $i=129.2^{\circ}$, the phase space becomes discontinuous and only scattered points are left without any recognizable dynamical structure (similar to the case for $1996 \mathrm{HW} 1$ at $i=143.2^{\circ}$ ). The reason will be explained later this section.

The situation is quite different for the prograde case, as shown in Fig. 10. It can be seen that $\mathcal{H}_{\text {reson } 2}$ is completely inside $\mathcal{H}_{\text {reson } 1}$ for all inclinations; also the width of $\mathcal{H}_{\text {reson } 2}$ increases as the orbit gets more inclined. When the strength of $\mathcal{H}_{\text {reson } 2}$ is very weak at $i=11.5^{\circ}$, a very tiny chaotic layer is present around the separatrix of $\mathcal{H}_{\text {reson } 1}$. When $\mathcal{H}_{\text {reson } 2}$ becomes stronger at $i=34.4^{\circ}$, new islands inside the two main libration regions are generated, in addition to the weak chaos. Finally, when $\mathcal{H}_{\text {reson } 2}$ is large enough at $i=45.8^{\circ}$, the original phase structure is broken and the two main islands are filled with chaos but are not connected anymore. Although the two resonances overlap completely, the generation of chaos is still closely related to the relative strengths of them. Combined with the above analysis, the extent of chaos is determined by both the location of the two resonances and their relative strength. The dynamics of $\mathcal{H}_{2 \text { dof }}$ is determined by the evolution (location, stability and strength) of both $\mathcal{H}_{\text {reson } 1}$ and $\mathcal{H}_{\text {reson } 2}$ as well as their interaction. 

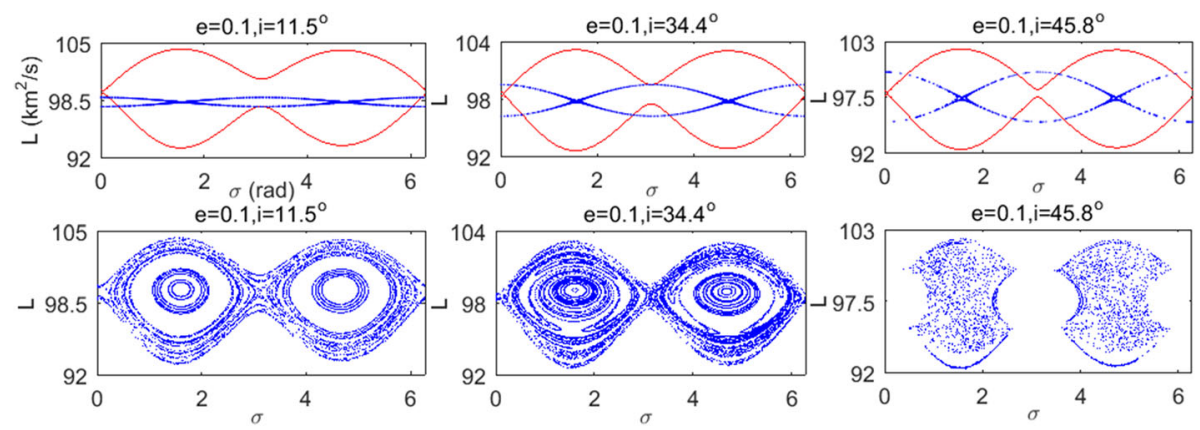

Fig. 10 First row the separatrices of resonances $\mathcal{H}_{\text {reson } 1}$ (red) and $\mathcal{H}_{\text {reson } 2}$ (blue) on the section $g=\pi / 2, \dot{g}>0$; Second row the phase space of the corresponding $\mathcal{H}_{2 \text { dof }}$; both for $e=0.1, i=$ $11.5^{\circ}, 34.4^{\circ}, 45.8^{\circ}$ (prograde)
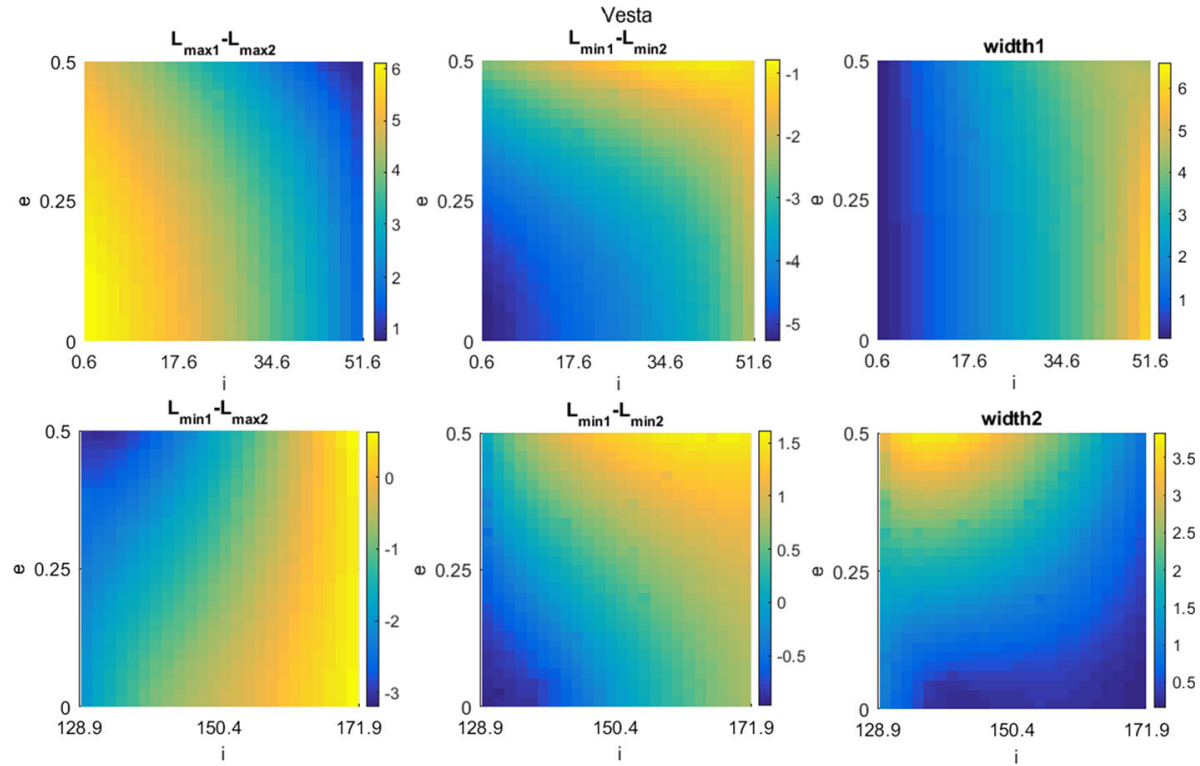

Fig. 11 The distance between $\mathcal{H}_{\text {reson } 1}$ and $\mathcal{H}_{\text {reson } 2}$ measured as $L_{\min 1}-L_{\max 2}$ (left) and $L_{\min 1}-L_{\min 2}$ (middle), and the width of $\mathcal{H}_{\text {reson } 2}$ (right) for the prograde (top) and retrograde (bottom) cases ( $i$ in radian)

\subsubsection{The effect of $e$}

Fig. 11 shows contour maps that can be used to analyze the impact of $e$. For the retrograde case, the effects of $e$ and $i$ on the evolution of the two resonances are similar to that of 1996 HW1, as shown in the bottom plots of Fig. 11. The slight difference is that the maximum resonance width of $\mathcal{H}_{\text {reson } 2}$ is at $e=0.5$ and $i \approx 137.5^{\circ}$ for Vesta rather than at the top-left corner for $1996 \mathrm{HW} 1$, which can be explained by the non-linear property of the resonance width as a function of $e$ and $i$. For the prograde case, instead of $L_{\min 1}-L_{\max 2}, L_{\max 1}-L_{\max 2}$ is obtained due to the fact that the two resonances already completely overlap. It is always positive for $L_{\max 1}-L_{\max 2}$ and negative for $L_{\min 1}-L_{\min 2}$. It must be mentioned that when $i=0$, the width of $\mathcal{H}_{\text {reson } 2}$ is zero and the dynamics of $\mathcal{H}_{\text {reson } 1}$ is not affected. Therefore, we start 


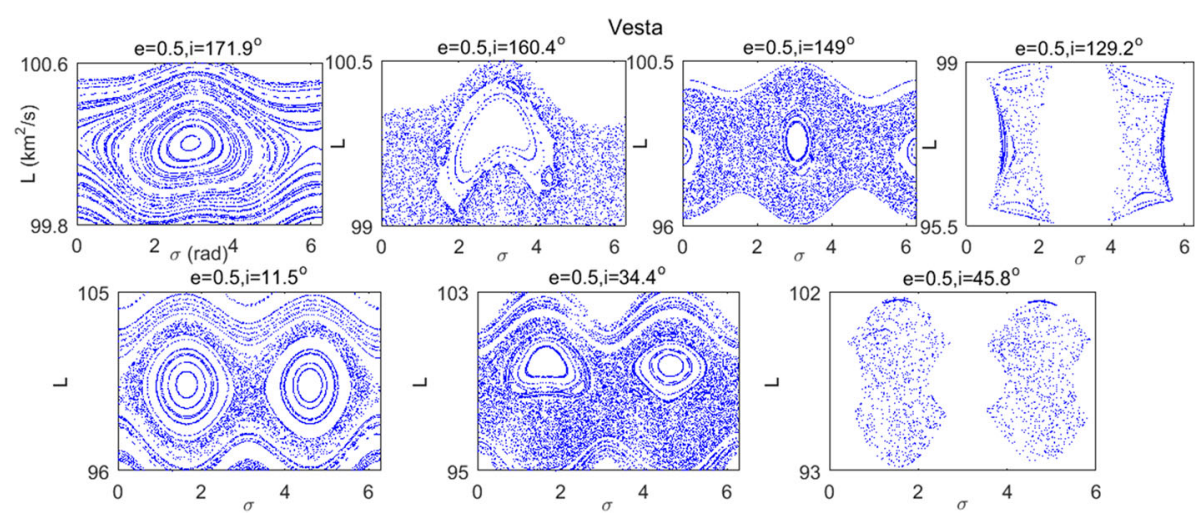

Fig. 12 The phase space of $\mathcal{H}_{2 \text { dof }}$ at $e=0.5$ for $i=171.9^{\circ}, 160.4^{\circ}, 149^{\circ}, 129.2^{\circ}, 11.5^{\circ}, 34.4^{\circ}, 45.8^{\circ}$

our calculation from $i=0.6^{\circ}$. The largest distances between the maximum and minimum boundaries of the two resonances are both at the left-bottom corner of the contour map, and the smallest distances between them at the right-top corner, which can be easily explained by the corresponding weakest and strongest perturbing effect of $\mathcal{H}_{\text {reson } 2}$. In addition, the width of $\mathcal{H}_{\text {reson } 2}$ achieves its largest value at the largest inclination but smallest eccentricity. It can be noticed that the ranges of $i$ stop at $51.6^{\circ}$ and $128.9^{\circ}$ for the prograde and retrograde orbits, respectively, due to the break of the separatrix of $\mathcal{H}_{\text {reson } 2}$ at $51.6^{\circ} \precsim i \precsim 128.9^{\circ}$.

For a complete understanding, the phase space of $\mathcal{H}_{2 \text { dof }}$ at $e=0.5$ is given in Fig. 12. Similarly, compared to the phase space at $e=0.1$ (shown in Figs. 9 and 10), the main island is strongly distorted and the chaotic region is significantly extended, due to the strong perturbation of $\mathcal{H}_{\text {reson } 2 .}$. For $e=0.5, i=149^{\circ}$, the regular region at the center of the phase space again is actually part of the regular region of $\mathcal{H}_{\text {reson } 2}$, due to the comparable influence of $\mathcal{H}_{\text {reson } 1}$ and $\mathcal{H}_{\text {reson } 2}$ on the dynamics of $\mathcal{H}_{2 \text { dof }}$. Therefore, a large $e$ gives rise to strong perturbations on the dynamics.

\subsubsection{Near polar region}

For the near polar region, the dynamical structure shrinks and almost disappears on our previously defined section, as can already be seen from the plots at $i=45.8^{\circ}$ and $i=129.2^{\circ}$ in Figs. 9 and 10. This is due to the fact that the secular rate of $g$ (Kaula 1966)

$$
\dot{g}=-\frac{3 n R^{2} C_{20}}{a^{2}\left(1-e^{2}\right)^{2}}\left(4-5 s^{2}\right)
$$

changes sign at the critical inclinations $i_{\text {critical }}=63.4^{\circ}$ and $116.6^{\circ}$. In particular, it is negative for $63.4^{\circ}<i<116.6^{\circ}$. Since this formula of $\dot{g}$ is obtained from averaging the leading $\mathrm{C}_{20}$ perturbation and our current model includes additional harmonics terms, the sign of $\dot{g}$ in our study does not change sharply at $i_{\text {critical }}$ but has a transition process. However, the exact values of this transition are beyond the scope of this study. Some orbits may still have $\dot{g}>0$ while others already have $\dot{g}<0$, which explains the break of the separatrix of $\mathcal{H}_{\text {reson } 2}$ on the section $g=\pi / 2, \dot{g}>0$. Therefore, we can define a new section for the near polar orbits with the only difference that $\dot{g}<0$.

Results are shown in Fig. 13. For the case of $e=0.1$, no proper solution of $\left(L^{*}, G^{*}\right)$ of $\mathcal{H}_{\text {reson } 2}$ can be found on this new section; and its Poincaré map rather than the separatrix is included. The two resonances have moderate overlap at the upper and lower boundaries of 

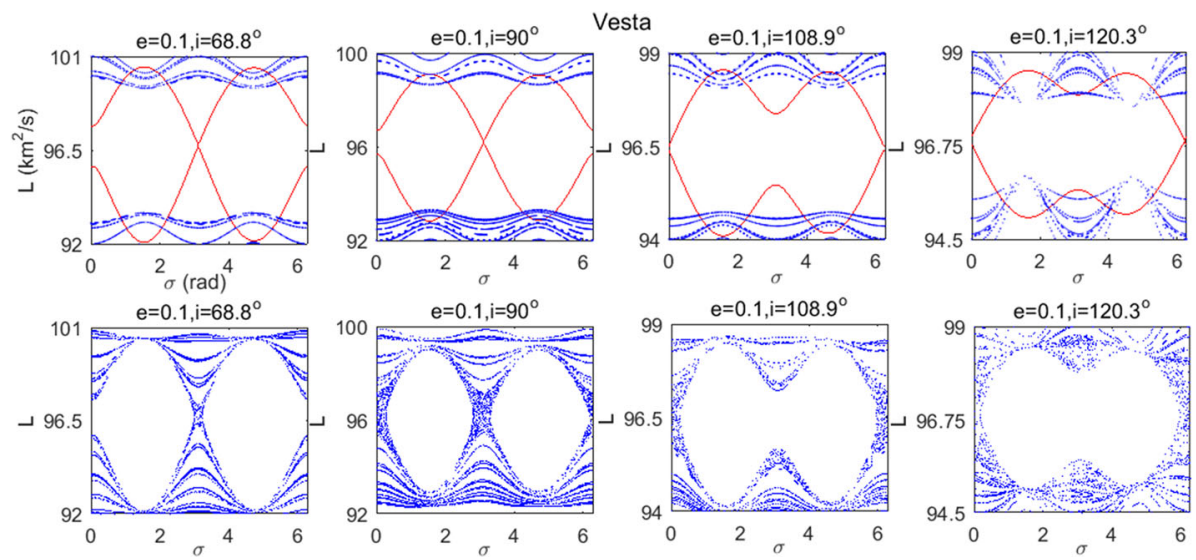

Fig. 13 First row the separatrices of resonances $\mathcal{H}_{\text {reson } 1}$ (red) and $\mathcal{H}_{\text {reson } 2}$ (blue) on the section $g=\pi / 2, \dot{g}<0$; second row the phase space of the corresponding $\mathcal{H}_{2 \text { dof }}$; both for $e=0.1, i=$ $68.8^{\circ}, 90^{\circ}, 108.9^{\circ}, 120.3^{\circ}$

$\mathcal{H}_{\text {reson } 1}$, which brings about limited chaotic regions closely attached to the separatrix. When $\mathcal{H}_{\text {reson } 2}$ reaches its strongest effect at $i=90^{\circ}$, the chaos becomes more obvious and thick. For $i=68.8^{\circ}$ and $108.9^{\circ}$, the chaos is visible but less abundant. For $i=120.3^{\circ}$, in addition to the chaos, islands are apparent in the circulation region of $\mathcal{H}_{\text {reson } 1}$, where the two resonances have a strong modulation with each other. However, with the increase of $e,\left(L^{*}, G^{*}\right)$ can be found and the separatrix of $\mathcal{H}_{\text {reson } 2}$ can be obtained on the new section. The example of $i=90^{\circ}$ at $e=0.3,0.5$ is given in Fig. 14A-D. It can be seen that for $e=0.3$, there is an overlap between the separatrices of the two Hamiltonians, which leads to the expansion of the chaos in the circulation region. When $e$ reaches 0.5 , the perturbing Hamiltonian $\mathcal{H}_{\text {reson } 2}$ becomes dominant, as can be seen from the width of the libration region in Fig. 14C, and resultantly the Poincaré map is the phase space of $\mathcal{H}_{\text {reson } 2}$ with chaotic regions generated by the perturbation of $\mathcal{H}_{\text {reson } 1 .}$. One more example is given for $i=100^{\circ}$. For the small value of $e=0.1$, the circulation region is filled with chaos. The chaos extends and regular libration region shrinks for $e=0.3$. When $e$ is large enough at 0.5 , the phase space is occupied by the perturbing Hamiltonian $\mathcal{H}_{\text {reson } 2 \text {. }}$

Therefore, for near polar orbits the libration region of $\mathcal{H}_{\text {reson } 1}$ is hardly influenced by $\mathcal{H}_{\text {reson } 2}$ for small eccentricities, in spite of the strong effects of the perturbations of $\mathcal{H}_{\text {reson } 2}$ at large eccentricities.

\subsection{Betulia}

For both prograde and retrograde orbits, Betulia has very similar properties as Vesta, concerning the distance between the two resonances and the width of $\mathcal{H}_{\text {reson } 2}$.

\subsubsection{The effect of $i$}

The retrograde case is illustrated in Fig. 15. Differently from Fig. 9, the phase space is not symmetric with respect to $i=90^{\circ}$ anymore. When the two resonances are further apart at $i=171.9^{\circ}, \mathcal{H}_{\text {reson } 1}$ is hardly influenced. When they are almost in contact with each other at $i=165^{\circ}$, thick chaotic layers are present together with small islands in the phase space. Before complete overlap, there is a small KAM-tori left at $i=153.6^{\circ}$ and the center region 

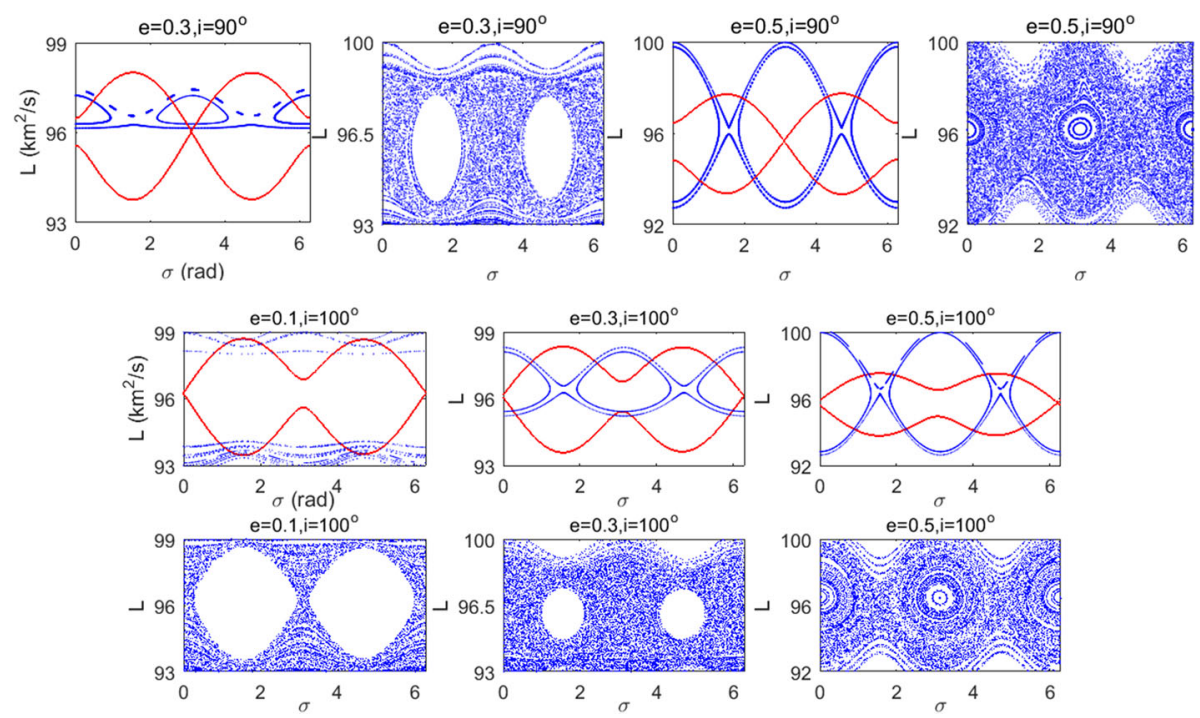

Fig. 14 First row the separatrices of $\mathcal{H}_{\text {reson } 1}($ red $)$ and $\mathcal{H}_{\text {reson } 2}$ (blue) and the corresponding $\mathcal{H}_{2 \text { dof }}$; for $i=90^{\circ}, e=0.3, .5$; Second and third rows the separatrices of $\mathcal{H}_{\text {reson } 1}$ (red) and $\mathcal{H}_{\text {reson } 2}$ (blue) and the corresponding $\mathcal{H}_{2 \text { dof }}$, respectively; for $i=100^{\circ}, e=0.1, .3, .5$

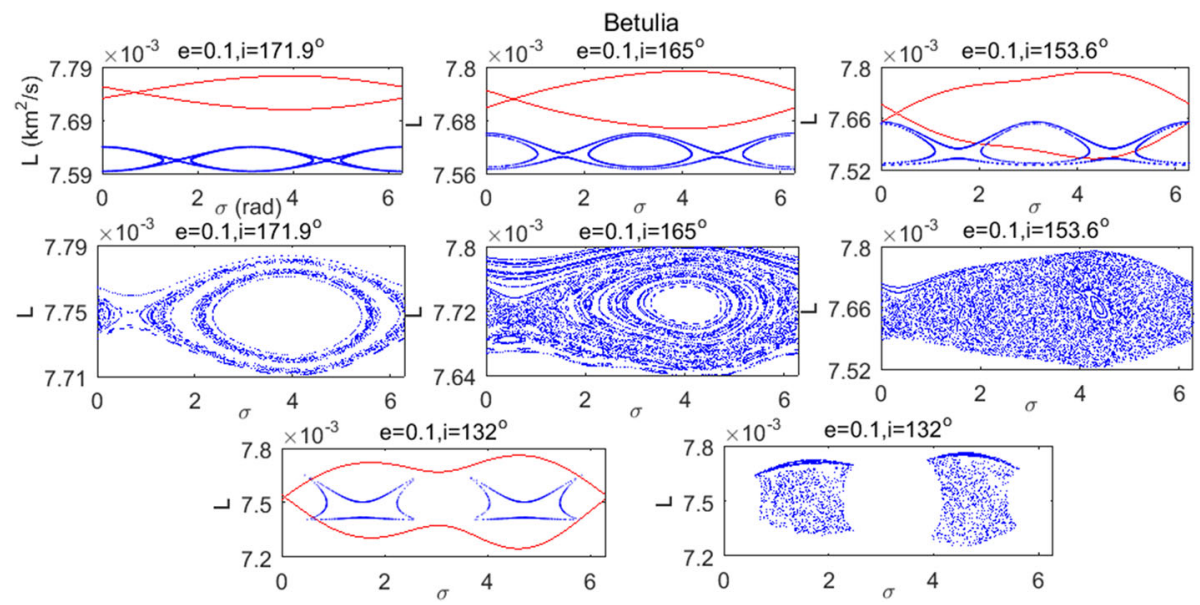

Fig. 15 First row the separatrices of resonances $\mathcal{H}_{\text {reson } 1}$ (red) and $\mathcal{H}_{\text {reson } 2}$ (blue) on the section $g=\pi / 2, \dot{g}>0$; second row the phase space of the corresponding $\mathcal{H}_{2 \text { dof }}$; both for $e=0.1, i=$ $171.9^{\circ}, 165^{\circ}, 153.6^{\circ}$; third row the separatrices and phase space for $e=0.1, i=132^{\circ}$ (retrograde)

of the phase space is also distorted. After that at $i=132^{\circ}$, the phase space is totally chaotic and finally broken.

For the prograde case, the two resonances always totally overlap, as shown in Fig. 16. When $\mathcal{H}_{\text {reson } 2}$ is tiny and weak at $i=11.5^{\circ}$, the main structure of $\mathcal{H}_{\text {reson } 1}$ is kept, with the difference that small chaotic regions appear near the separatrix and again new islands are generated inside the right main island. As $\mathcal{H}_{\text {reson } 2}$ becomes stronger at $i=34.4^{\circ}$, the overlapping part of $\mathcal{H}_{\text {reson } 1}$ is completely chaotic. Similarly, for $i=45.8^{\circ}$, the phase space 

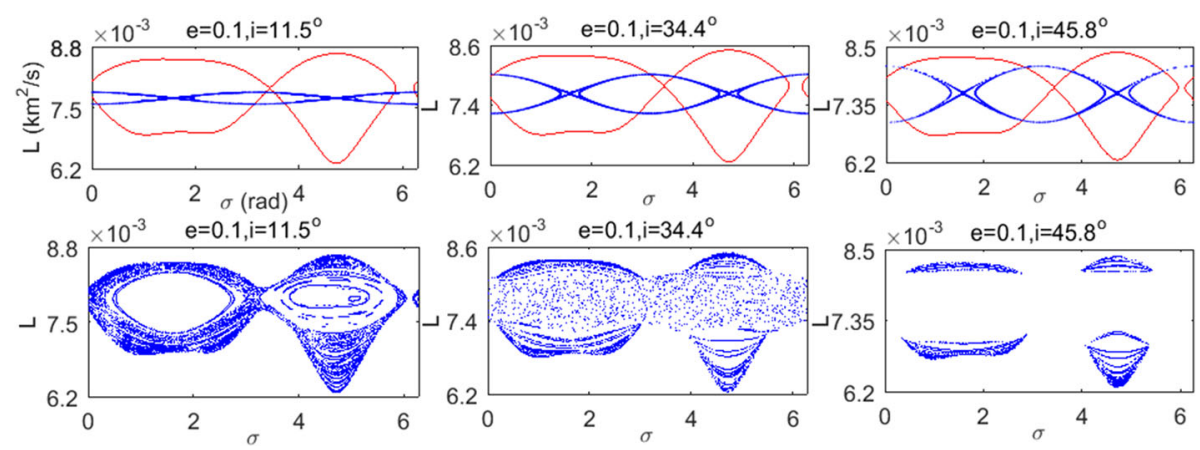

Fig. 16 First row the separatrices of resonances $\mathcal{H}_{\text {reson } 1}($ red $)$ and $\mathcal{H}_{\text {reson } 2}($ blue $)$ on the section $g=$ $\pi / 2, \dot{g}>0 ;$ second row the phase space of the corresponding $\mathcal{H}_{2 \text { dof }}$; both for $e=0.1, i=11.5^{\circ}, 34.4^{\circ}, 45.8^{\circ}$ (prograde)
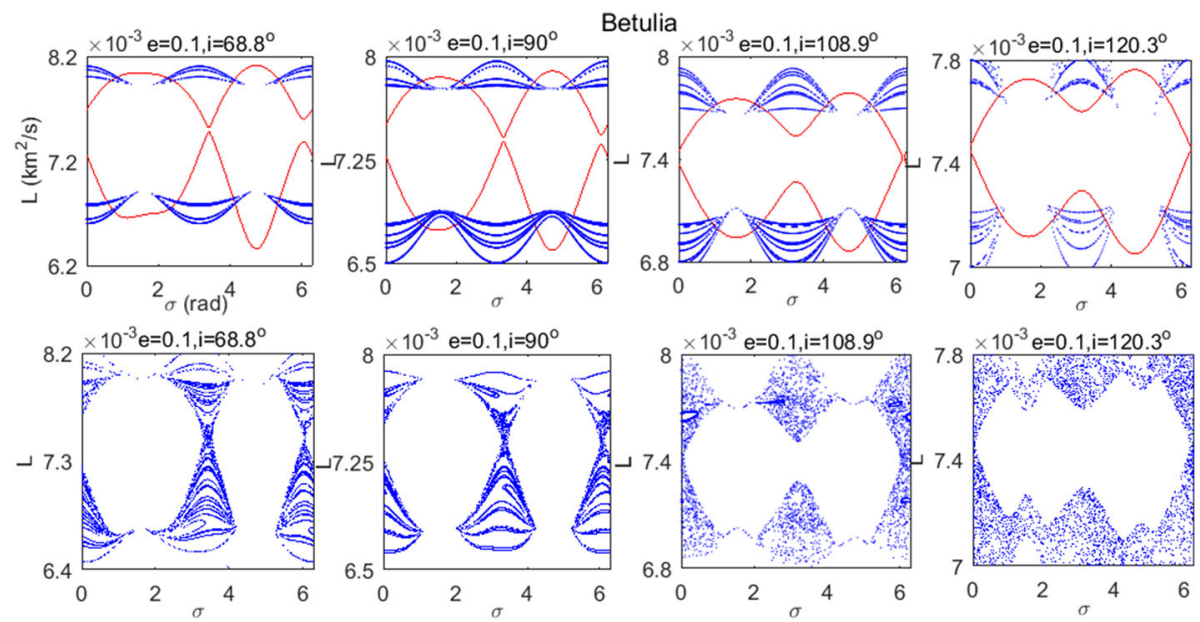

Fig. 17 First row the separatrices of resonances $\mathcal{H}_{\text {reson } 1}$ and $\mathcal{H}_{\text {reson } 2}$ on the section $g=\pi / 2, \dot{g}<0$; second row the phase space of the corresponding $\mathcal{H}_{2 \text { dof }}$; both for $e=0.1, i=68.8^{\circ}, 90^{\circ}, 108.9^{\circ}, 120.3^{\circ}$

of $\mathcal{H}_{\text {reson } 1}$ is significantly broken and is left with large gap regions, even without complete break of the separatrix of $\mathcal{H}_{\text {reson } 2}$. This indicates the strong perturbation of $\mathcal{H}_{\text {reson } 2}$ on the dynamics and the highly non-linear property of $\mathcal{H}_{2 \text { dof }}$.

For the near polar region, as illustrated in Fig. 17, Betulia has a similar property as Vesta, considering that chaotic layers appear in the vicinity of the separatrix and also new islands are generated in the circulation region. However, for Betulia the three regions are weakly connected at $i=68.8^{\circ}$. Furthermore, they become totally isolated at $i=90^{\circ}$, due to the stronger modulation of $\mathcal{H}_{\text {reson } 2}$ compared to that of Vesta (Fig. 13). Since the regular region is open, the originally stable EPs of $\mathcal{H}_{\text {reson } 1}$ probably change into unstable. At $i=108.9^{\circ}$ and $120.3^{\circ}$, the circulation region is full of chaos, which implies that the perturbation of $\mathcal{H}_{\text {reson } 2}$ and its interaction with $\mathcal{H}_{\text {reson } 1}$ in this region are stronger, compared to the cases at $i=68.8^{\circ}$ and $90^{\circ}$. Again, the general structure of the libration part of $\mathcal{H}_{\text {reson } 1}$ is kept. 


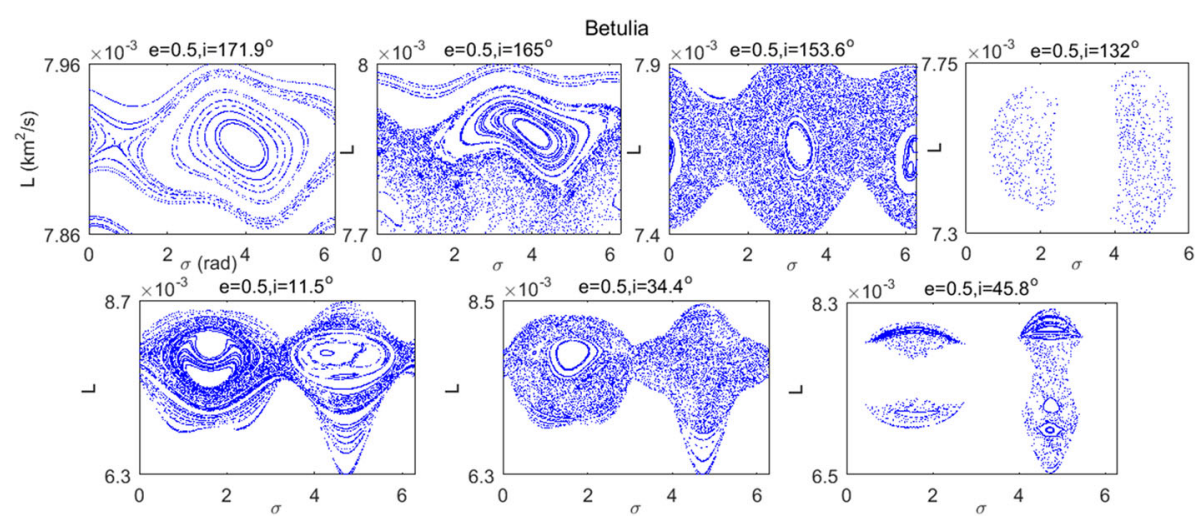

Fig. 18 The phase space of $\mathcal{H}_{2 \text { dof }}$ at $e=0.5$ for $i=171.9^{\circ}, 165^{\circ}, 153.6^{\circ}, 132^{\circ}, 11.5^{\circ}, 34.4^{\circ}, 45.8^{\circ}$

\subsubsection{The effect of $e$}

In addition, it is also found that $e$ shows the same effect on the dynamics of Betulia as for Vesta, both for the prograde and retrograde orbits. The mechanism is the same and is not explained in detail here. However, the phase space of $\mathcal{H}_{2 d o f}$ at $e=0.5$ is included in Fig. 18. For the first plot at $i=171.9^{\circ}$, the main island is highly distorted although still without chaos. For $i=165^{\circ}$, the distortion is more serious and large chaos appears. The phase space with a small area of regular region in the center already shows the property of $\mathcal{H}_{\text {reson } 2}$ at $i=153.6^{\circ}$. For $i=11.5^{\circ}, 34.4^{\circ}, 45.8^{\circ}$, the chaotic region is extended and new structures are generated.

\section{The maximal Lyapunov Characteristic Exponent of chaotic orbits}

In addition to the above study about the extent of chaotic layers, the chaos can also be characterized quantitatively by calculating the value of the maximal Lyapunov Characteristic Exponent (mLCE), which is an indicator of the regular or chaotic properties of orbits (Skokos 2010). Its basic idea is to measure the distance between two orbits that start close, until infinite time $(t \rightarrow \infty)$. It characterizes the average growth rate of a small perturbation of the solution of a dynamical system and is defined as

$$
\lambda=\lim _{\mathrm{t} \rightarrow \infty} \frac{1}{t} \sum_{0}^{t} \ln \|\boldsymbol{v}(t)\|
$$

in which $\boldsymbol{v}(t)$ is the deviation vector with respect to the given orbit at time $t$. It is also the solution of the corresponding variational equations of the dynamical system. If $\lambda>0$, the orbit is chaotic; if $\lambda=0$, the orbit is regular. The numerical algorithm applied here is the standard method originally developed by Benettin and Galgani (1979). Its detailed implementation can be found in Skokos (2010). It has to be mentioned that for regular orbits it might take a long time for $\lambda$ to achieve zero. However, within a moderate time interval the tendency to zero is already visible.

Since it is obvious that large $e$ introduces stronger chaos and the chaos of the three different asteroids is expected to be compared, the mLCE of orbits selected from the chaotic and regular 

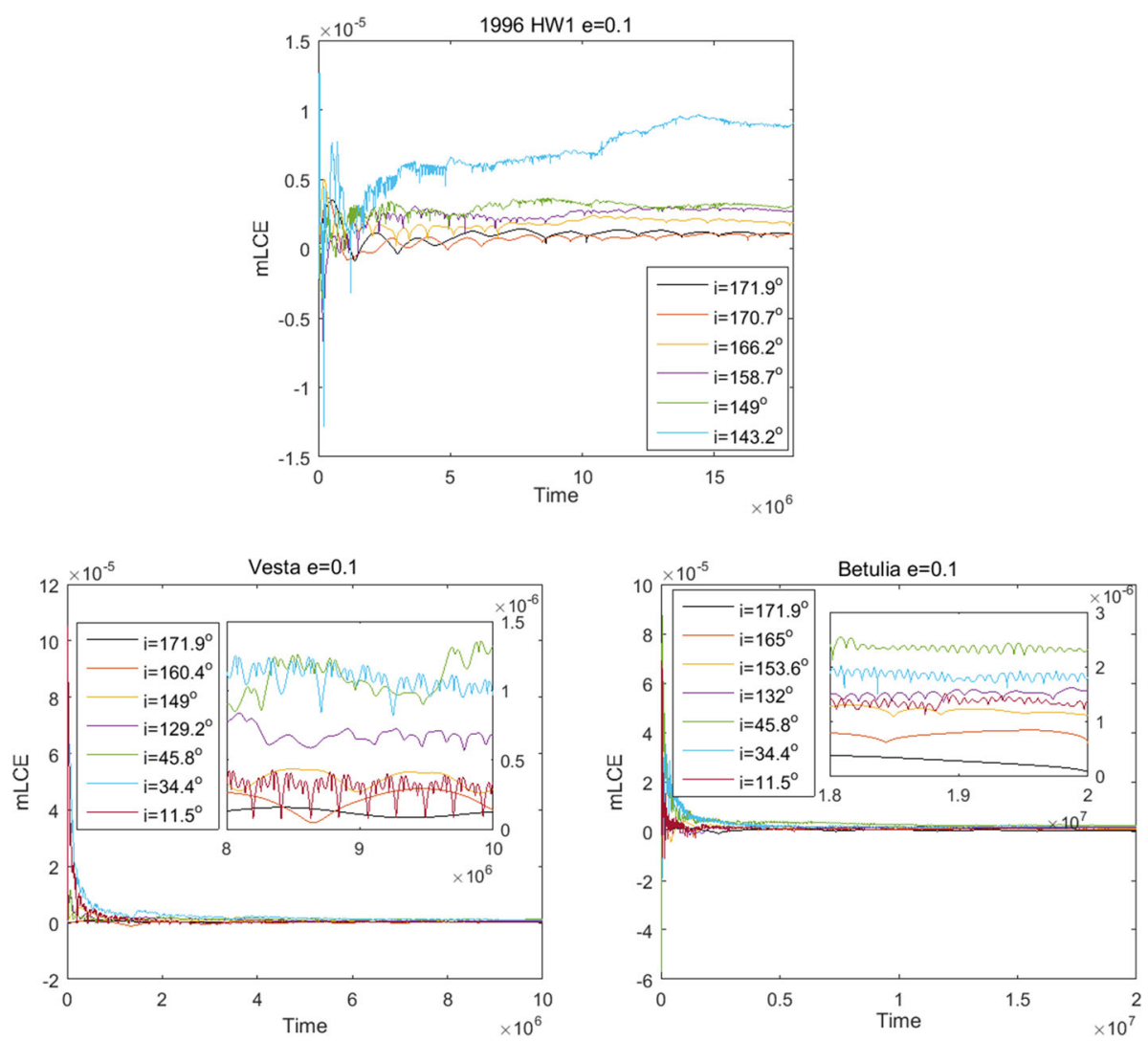

Fig. 19 The mLCE of regular and chaotic orbits from the Poincaré maps of $\mathcal{H}_{2}$ dof for 1996 HW1, Vesta and Betulia

regions (if there is no chaos) on the maps from Figs. 5, 9, 10, 12 and 13 are given in Fig. 19. These maps primarily indicate the effect of $i$ on dynamics at $e=0.1$. The total integration time for them is different, but has been chosen such that a stable value of all the mLCE values can be achieved. To make the results more visible, the mLCE values at the end of the integrations are magnified and are shown respectively as insets in the plots of Vesta and Betulia.

For the three asteroids, they share the same property that the more inclined the orbit, the larger the mLCE value, indicating the stronger chaotic property. In addition, the mLCE values of the retrograde orbits are generally smaller than those of the prograde ones. For $i=171.9^{\circ}$, there is no chaos on both the maps of Vesta and Betulia (shown in Figs. 5, 9). This is demonstrated in the value for the mLCE illustrated as black at the bottom of the inset which will finally tend to zero, the tendency of which can already be identified. The difference among the three asteroids can also be noticed. The resonant orbits around 1996 HW1 have the largest mLCE (at magnitude $10^{-5}$ ), the ones for Betulia rank second (at magnitude $10^{-6}$ ), while orbits around Vesta show the smallest mLCE (mostly at magnitude $10^{-7}$ ). This can be explained by the different values of $\mathrm{C}_{20}$ and $\mathrm{C}_{22}$ induced from the irregular shape of the body. The more irregular the gravitational field is, the larger the values of $\mathrm{C}_{20}$ and $\mathrm{C}_{22}$ and the impact of $\mathcal{H}_{\text {reson } 2}$ are. 


\section{Conclusions}

In this study, a 2-DOF Hamiltonian of the 1:1 ground-track resonance of a gravitational field up to degree and order 4 was built. The dominant part of the Hamiltonian, i.e. $\mathcal{H}_{0}$, was first studied by finding the EPs and examining their stability for non-circular and non-polar orbits of Vesta, $1996 \mathrm{HW} 1$ and Betulia. This $\mathcal{H}_{0}$ was proven to largely capture the dynamical characteristics of the 1:1 resonant dynamics for the three study cases. In particular, the inclination $i$ was found to play a significant role on the number of EPs: when $i$ approaches $\pi$, there is only one stable EP left, due to the dominant strength of $\mathrm{C}_{31}$ over $\mathrm{C}_{22}$ on the structure of the phase space. The 2nd degree and order harmonics largely determine the stability of the EP, while the higher order terms either introduce new EPs and change the resonance width or break the symmetry of the dynamics.

By applying Poincaré maps, the 2-DOF Hamiltonian $\mathcal{H}_{2}$ dof was then investigated. Two Hamiltonians $\mathcal{H}_{\text {reson } 1}$ and $\mathcal{H}_{\text {reson } 2}$ were defined in this 2 -DOF model and their locations and widths were determined numerically for different combinations of $e$ and $i$.

With the overlap criteria, the extent of chaotic regions was qualitatively explained by the distance between the two resonances as well as their resonance strength. For near-circular, near-equatorial orbits, the dynamics of $\mathcal{H}_{\text {reson } 1}$ around the stable EP is hardly influenced by the second resonance as $\mathcal{H}_{\text {reson } 1}$ and $\mathcal{H}_{\text {reson } 2}$ are further apart. When $i$ gets further away from the equatorial plane, $\mathcal{H}_{\text {reson } 2}$ becomes close to and almost interacts with $\mathcal{H}_{\text {reson } 1}$. Small-scale chaos was generated in the vicinity of the separatrix of $\mathcal{H}_{2 \text { dof }}$, whose boundaries were well estimated by the modulated-pendulum approximation. When the two resonances have an obvious overlap for $i$ getting close to the polar region, large chaos became apparent and new islands came forth in the phase space. However, for the near polar case at small eccentricities, the libration region of $\mathcal{H}_{\text {reson } 1}$ is hardly influenced and is stable against perturbation of

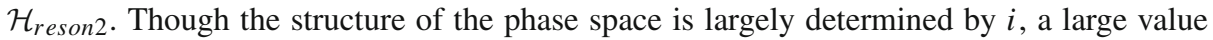

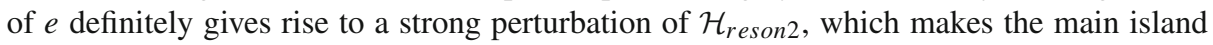
distorted and the chaotic region extended. Therefore, the retrograde, near polar and near circular orbits show the more stability against external perturbations.

In addition, the mLCEs of the chaotic and regular orbits were calculated, thereby confirming that the strongest chaos has to be expected in the vicinity of the most irregular gravitational field, e.g., 1996 HW1 in this study.

The results and analyses in this paper provide us insight on the impact of eccentricity and inclination, as well as the gravitational field, on the 1:1 ground track resonance. Through the study of overlap of resonances, the generation and width of chaos in the phase space of the 1:1 resonance can be estimated. For future study, the possibilities of capture into and escape from the 1:1 resonance should be addressed, which helps to control the spacecraft from chaotic motion during its transition through the resonance.

Acknowledgements We give our thanks to the anonymous reviewers for their comments to improve the quality of this paper. This research is funded by the Astrodynamics and Space Missions group, Delft University of Technology, The Netherlands and the China Scholarship Council. X. Hou also thanks the support from the National Science Foundation of China (11322330).

Open Access This article is distributed under the terms of the Creative Commons Attribution 4.0 International License (http://creativecommons.org/licenses/by/4.0/), which permits unrestricted use, distribution, and reproduction in any medium, provided you give appropriate credit to the original author(s) and the source, provide a link to the Creative Commons license, and indicate if changes were made. 


\section{Appendix 1}

See Table 1.

The expressions of $\mathcal{H}_{1}$ and $\mathcal{H}_{2}$ are given as

$$
\begin{aligned}
\mathcal{H}_{1}= & -\frac{\mu^{4} R^{2}}{L^{6}}\left[F_{210} G_{20-1}\left(C_{21} \cos (\sigma+g)+S_{21} \sin (\sigma+g)\right)\right. \\
& \left.+F_{211} G_{211}\left(C_{21} \cos (\sigma-g)+S_{21} \sin (\sigma-g)\right)\right] \\
& -\frac{\mu^{5} R^{3}}{L^{8}}\left[F_{301} G_{31-1}\left(C_{30} \cos g+S_{30} \sin g\right)+F_{302} G_{321}\left(C_{30} \cos g-S_{30} \sin g\right)\right] \\
& -\frac{\mu^{6} R^{4}}{L^{8}}\left[F_{411} G_{41-1}\left(C_{41} \cos (\sigma+g)+S_{41} \sin (\sigma+g)\right)\right. \\
& +F_{412} G_{421}\left(C_{41} \cos (\sigma-g)+S_{41} \sin (\sigma-g)\right)+F_{430} G_{40-1}\left(C_{43} \cos (3 \sigma+g)\right. \\
& \left.\left.+S_{43} \sin (3 \sigma+g)\right)+F_{431} G_{411}\left(C_{43} \cos (3 \sigma-g)+S_{43} \sin (3 \sigma-g)\right)\right] \\
\mathcal{H}_{2}= & -\frac{\mu^{4} R^{2}}{L^{6}}\left[F_{221} G_{212}\left(C_{22} \cos (2 \sigma-2 g)+S_{22} \sin (2 \sigma-2 g)\right)\right] \\
& -\frac{\mu^{5} R^{3}}{L^{8}}\left[F_{310} G_{30-2}\left(C_{31} \cos (\sigma+2 g)+S_{31} \sin (\sigma+2 g)\right)\right. \\
& +F_{312} G_{322}\left(C_{31} \cos (\sigma-2 g)+S_{31} \sin (\sigma-2 g)\right) \\
& \left.+F_{331} G_{312}\left(C_{33} \cos (3 \sigma-2 g)+S_{33} \sin (3 \sigma-2 g)\right)\right] \\
& -\frac{\mu^{6} R^{4}}{L^{10}}\left[F_{401} G_{41-2}\left(C_{40} \cos (2 g)+S_{40} \sin (2 g)\right)\right. \\
& \left.+F_{422} G_{422}\left(C_{42} \cos (2 \sigma-2 g)+S_{42} \sin (2 \sigma-2 g)\right)\right]
\end{aligned}
$$

Table 1 The primary zonal and tesseral terms contributing to the $1: 1$ resonance

\begin{tabular}{llllllll}
\hline$n$ & 2 & 2 & 3 & 3 & 4 & 4 & 4 \\
\hline$m$ & 0 & 2 & 1 & 3 & 0 & 2 & 4 \\
$p$ & 1 & 0 & 1 & 0 & 2 & 1 & 0 \\
$q$ & 0 & 0 & 0 & 0 & 0 & 0 & 0 \\
$\Theta_{n m p q}$ & 0 & $2 \sigma$ & $\sigma$ & $3 \sigma$ & 0 & $2 \sigma$ & $4 \sigma$ \\
\hline
\end{tabular}

\section{Appendix 2}

See Fig. 20.

The tables below contain the values for the un-normalized spherical harmonic coefficients to degree and order 4 for Vesta derived from Tricarico and Sykes (2010), 1996 HW1 (Feng et al. 2015a) and Betulia derived from Magri et al. (2011). Although there is an update of the gravitational field of Vesta in Konopliv et al. (2014), the full $4 \times 4$ spherical harmonics are not directly available from it. In addition, the difference between the two is quite small. 

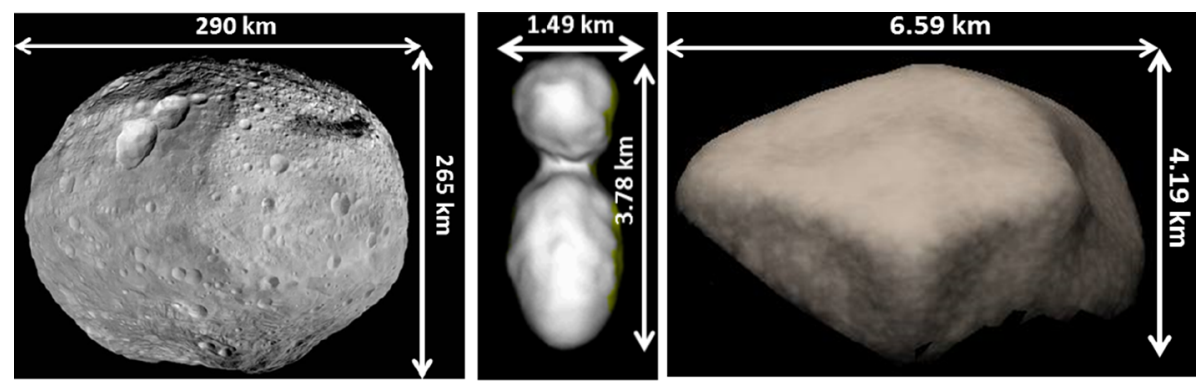

Fig. 20 The shape models of Vesta (left), image credit: NASA/DAWN; 1996 HW1 (middle) (Magri et al. 2011) and Betulia (right) (Magri et al. 2007)

\begin{tabular}{llllll}
\hline Vesta & \multicolumn{1}{l}{} \\
\hline $\mathrm{C}_{20}$ & $-6.872555 \times 10^{-2}$ & $\mathrm{~S}_{31}$ & $1.825409 \times 10^{-4}$ & $\mathrm{~S}_{41}$ & $-1.347130 \times 10^{-4}$ \\
$\mathrm{C}_{21}$ & 0 & $\mathrm{C}_{32}$ & $-3.162892 \times 10^{-4}$ & $\mathrm{C}_{42}$ & $-3.152856 \times 10^{-5}$ \\
$\mathrm{~S}_{21}$ & 0 & $\mathrm{~S}_{32}$ & $5.943231 \times 10^{-5}$ & $\mathrm{~S}_{42}$ & $6.551679 \times 10^{-5}$ \\
$\mathrm{C}_{22}$ & $3.079667 \times 10^{-3}$ & $\mathrm{C}_{33}$ & $2.565757 \times 10^{-5}$ & $\mathrm{C}_{43}$ & $-3.113571 \times 10^{-5}$ \\
$\mathrm{~S}_{22}$ & 0 & $\mathrm{~S}_{33}$ & $7.264998 \times 10^{-5}$ & $\mathrm{~S}_{43}$ & $-2.689264 \times 10^{-6}$ \\
$\mathrm{C}_{30}$ & $6.286305 \times 10^{-3}$ & $\mathrm{C}_{40}$ & $9.6 \times 10^{-3}$ & $\mathrm{C}_{44}$ & $3.190457 \times 10^{-6}$ \\
$\mathrm{C}_{31}$ & $-7.982112 \times 10^{-4}$ & $\mathrm{C}_{41}$ & $6.394125 \times 10^{-4}$ & $\mathrm{~S}_{44}$ & $5.514632 \times 10^{-6}$ \\
\hline
\end{tabular}

1996 HW1 (all $\mathrm{S}_{\mathrm{nm}}$ terms are zero)

\begin{tabular}{llllll}
\hline $\mathrm{C}_{20}$ & $-1.21847 \times 10^{-1}$ & $\mathrm{C}_{31}$ & $-1.3964 \times 10^{-2}$ & $\mathrm{C}_{41}$ & 0 \\
$\mathrm{C}_{21}$ & 0 & $\mathrm{C}_{32}$ & 0 & $\mathrm{C}_{42}$ & $-4.258 \times 10^{-3}$ \\
$\mathrm{C}_{22}$ & $5.8547 \times 10^{-2}$ & $\mathrm{C}_{33}$ & $2.547 \times 10^{-3}$ & $\mathrm{C}_{43}$ & 0 \\
$\mathrm{C}_{30}$ & 0 & $\mathrm{C}_{40}$ & $3.8779 \times 10^{-2}$ & $\mathrm{C}_{44}$ & $5.16 \times 10^{-4}$ \\
\hline
\end{tabular}

\section{Betulia}

\begin{tabular}{llllll}
\hline $\mathrm{C}_{20}$ & $-1.476131 \times 10^{-1}$ & $\mathrm{~S}_{31}$ & $-2.491845 \times 10^{-3}$ & $\mathrm{~S}_{41}$ & $-5.428366 \times 10^{-4}$ \\
$\mathrm{C}_{21}$ & 0 & $\mathrm{C}_{32}$ & $-5.879324 \times 10^{-3}$ & $\mathrm{C}_{42}$ & $-1.599034 \times 10^{-3}$ \\
$\mathrm{~S}_{21}$ & 0 & $\mathrm{~S}_{32}$ & $2.931994 \times 10^{-3}$ & $\mathrm{~S}_{42}$ & $5.556629 \times 10^{-5}$ \\
$\mathrm{C}_{22}$ & $1.711891 \times 10^{-2}$ & $\mathrm{C}_{33}$ & $3.182376 \times 10^{-4}$ & $\mathrm{C}_{43}$ & $1.775273 \times 10^{-4}$ \\
$\mathrm{~S}_{22}$ & 0 & $\mathrm{~S}_{33}$ & $-3.910856 \times 10^{-3}$ & $\mathrm{~S}_{43}$ & $2.49498 \times 10^{-4}$ \\
$\mathrm{C}_{30}$ & $9.543225 \times 10^{-3}$ & $\mathrm{C}_{40}$ & $4.2618 \times 10^{-2}$ & $\mathrm{C}_{44}$ & $-3.298214 \times 10^{-5}$ \\
$\mathrm{C}_{31}$ & $-2.738977 \times 10^{-3}$ & $\mathrm{C}_{41}$ & $-6.251823 \times 10^{-4}$ & $\mathrm{~S}_{44}$ & $3.024807 \times 10^{-5}$ \\
\hline
\end{tabular}

\section{Appendix 3}

The location of EPs and resonance width at different combinations of $e$ and $i$ for Vesta, 1996 HW1 and Betulia. 

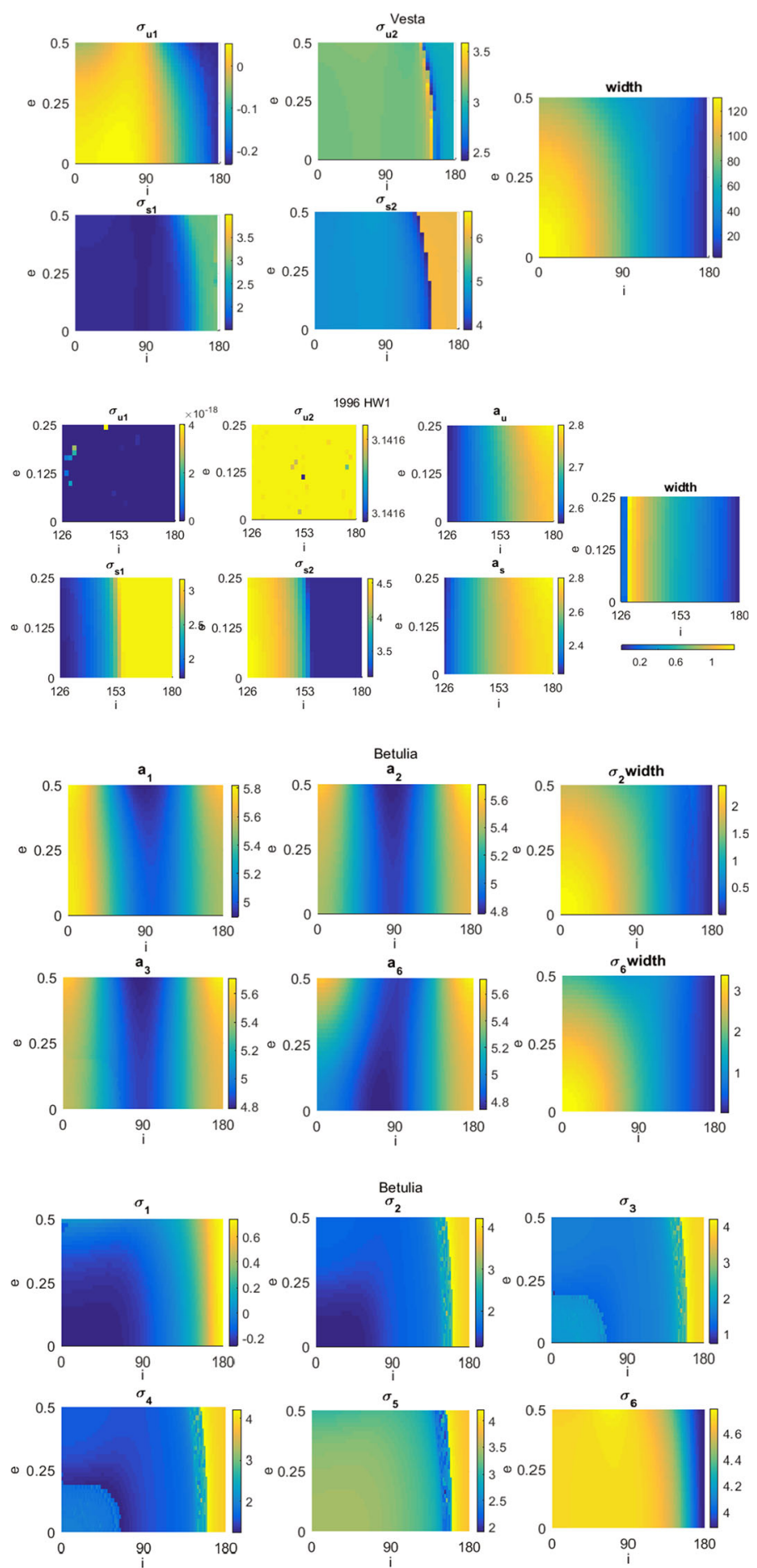


\section{References}

Benettin, G., Galgani, L.: Lyapunov characteristic exponents and stochasticity. Cargese, (1979)

Chao, C.-C.: Applied orbit perturbation and maintenance, Amer Inst of Aeronautics \& (2005)

Chirikov, B.V.: A universal instability of many-dimensional oscillator systems. Phys. Rep. 52, 263-379 (1979)

Compère, A., Lemaître, A., Delsate, N.: Detection by MEGNO of the gravitational resonances between a rotating ellipsoid and a point mass satellite. Celest. Mech. Dyn. Astron. 112, 75-98 (2012)

Delhaise, F., Henrard, J.: The problem of critical inclination combined with a resonance in mean motion in artificial satellite theory. Celest. Mech. Dyn. Astron. 55, 261-280 (1993)

Delsate, N.: Analytical and numerical study of the ground-track resonances of Dawn orbiting Vesta. Planet. Space Sci. 59, 1372-1383 (2011)

Feng, J., Noomen, R., Visser, P.N., Yuan, J.: Modeling and analysis of periodic orbits around a contact binary asteroid. Astrophys. Space Sci. 357, 1-18 (2015a)

Feng, J., Noomen, R., Yuan, J.: Orbital Motion in the Vicinity of the Non-collinear Equilibrium Points of a Contact Binary Asteroid. Planet. Space Sci. 117, 1-14 (2015b)

Henrard, J.: A semi-numerical perturbation method for separable Hamiltonian systems. Celest. Mech. Dyn. Astron. 49, 43-67 (1990)

Hu, W., Scheeres, D.: Numerical determination of stability regions for orbital motion in uniformly rotating second degree and order gravity fields. Planet. Space Sci. 52, 685-692 (2004)

Kaula, W.M.: Theory of satellite geodesy: applications of satellites to geodesy. Blaisdell Publishing Company, Massachusetts (1966)

Konopliv, A., Asmar, S., Park, R., Bills, B., Centinello, F., Chamberlin, A., et al.: The Vesta gravity field, spin pole and rotation period, landmark positions, and ephemeris from the Dawn tracking and optical data. Icarus 240, 103-117 (2014)

Magri, C., Howell, E.S., Nolan, M.C., Taylor, P.A., Fernández, Y.R., Mueller, M., et al.: Radar observations and a physical model of Asteroid 1580 Betulia. Icarus, 186, 152-177 (2007)

Magri, C., Ostro, S.J., Scheeres, D.J., Nolan, M.C., Giorgini, J.D., Benner, L.A.M., et al.: Radar observations and a physical model of Asteroid 1580 Betulia. Icarus 186, 152-177 (2007)

Morbidelli, A.: Modern celestial mechanics: aspects of solar system dynamics. Taylor \& Francis, London (2002)

Olsen, Ø.: Orbital resonance widths in a uniformly rotating second degree and order gravity field. Astron. Astrophys. 449, 821-826 (2006)

Scheeres, D.: Dynamics about uniformly rotating triaxial ellipsoids: applications to asteroids. Icarus 110, 225-238 (1994)

Scheeres, D., Ostro, S., Hudson, R., Werner, R.: Orbits close to asteroid 4769 Castalia. Icarus 121, 67-87 (1996)

Skokos, C.: The Lyapunov characteristic exponents and their computation. Dynamics of small solar system bodies and exoplanets. Springer, Berlin (2010)

Tricarico, P., Sykes, M.V.: The dynamical environment of Dawn at Vesta. Planet. Space Sci. 58, 1516-1525 (2010)

Tzirti, S., Varvoglis, H.: Motion of an Artificial Satellite around an Asymmetric, Rotating Celestial Body: Applications to the Solar System. PhD dissertation, Aristotle University of Thessaloniki (2014)

Valk, S., Lemaître, A., Deleflie, F.: Semi-analytical theory of mean orbital motion for geosynchronous space debris under gravitational influence. Adv. Space Res. 43, 1070-1082 (2009) 